\title{
Psycho-

\section{Specific impairments in instrumental learning following chronic intermittent toluene inhalation in adolescent rats}

\begin{tabular}{|r|l|}
\hline Journal: & Psychopharmacology \\
\hline Manuscript ID: & Psych-2013-00466.R2 \\
\hline Manuscript Type: & Original Investigation \\
\hline Date Submitted by the Author: & n/a \\
\hline Complete List of Authors: & $\begin{array}{l}\text { Dick, Alec; Florey Institute of Neuroscience and Mental Health, Behavioural } \\
\text { Neuroscience } \\
\text { Axelsson, Martin; Florey Institute of Neuroscience and Mental Health, } \\
\text { Behavioural Neuroscience } \\
\text { Lawrence, Andrew; Florey Institute of Neuroscience and Mental Health, } \\
\text { Behavioural Neuroscience } \\
\text { Duncan, Jhodie; Florey Institute of Neuroscience and Mental Health, } \\
\text { Behavioural Neuroscience }\end{array}$ \\
\hline Keywords: & COGNITION, ADDICTION, ABUSE, SELF-ADMINISTRATION \\
\hline \multicolumn{2}{|c}{} \\
\hline
\end{tabular}

SCHOLARONE ${ }^{\text {m }}$

Manuscripts 
Title: Specific impairments in instrumental learning following chronic intermittent toluene inhalation in adolescent rats

\author{
Alec L.W. Dick ${ }^{1,2}$, Martin Axelsson ${ }^{1,4}$, Andrew J. Lawrence ${ }^{1,2} \&$ Jhodie R. Duncan ${ }^{1,3}$ \\ ${ }^{1}$ Florey Institute of Neuroscience \& Mental Health; ${ }^{2}$ Florey Department of Neuroscience \\ and Mental Health; ${ }^{3}$ Department Anatomy \& Neuroscience, University of Melbourne, \\ Parkville, Victoria, 3010; ${ }^{4}$ Department of Applied Psychology, Royal Melbourne University \\ of Technology, Melbourne, Victoria, 3000, Australia
}

\title{
Communicating Authors:
}

Dr Jhodie Duncan or Professor Andrew Lawrence

Florey Institute of Neuroscience \& Mental Health,

L3, Kenneth Myer Building

Cnr Genetics Lane on Royal Parade

University of Melbourne, Parkville, Victoria, 3010, Australia

Telephone: +613 9035 6731/6692

Fax: +61309353107

Email address:

Jhodie.duncan@florey.edu.au or Andrew.Lawrence@florey.edu.au

\section{Funding:}

This study was supported by The National Health and Medical Research Council of Australia of which AJL is a Principal Research Fellow (1020737), the Australian Research Council (DP 110100379) of which JRD is a Future Fellow (100100235) and the Victorian Government's Operational Infrastructure Support Scheme. There are no conflicts of interest in this study. 


\begin{abstract}
Rationale Inhalant abuse is prevalent in adolescent populations, with chronic use resulting in neurobiological and cognitive abnormalities in adulthood. However, the nature and persistence of cognitive dysfunction, particularly following adolescent inhalant abuse, remains equivocal. Objective The present study assessed specific cognitive processes beginning in late adolescence and adulthood following adolescent inhalation of toluene, a main component of many compounds readily abused. Methods Adolescent male Wistar rats (postnatal day (PN) 27) were exposed to chronic intermittent inhaled toluene (10,000 ppm) for $1 \mathrm{hr} /$ day, 3 days/week for 4 weeks (PN 27 - 52) to mimic the patterns observed in human adolescent inhalant abusers. Following toluene exposure, motor and cognitive function was assessed. Results Adolescent toluene exposure did not alter motor learning in the Rotarod task (PN 58), or acquisition, reversal or retention of spatial learning in the Morris water maze (PN 55 - 64). In contrast, it delayed acquisition of instrumental responding for sucrose $(5 \% \mathrm{w} / \mathrm{v})$, and impaired operant reversal learning and cue-induced reinstatement of sucrose-seeking in adulthood (PN $57-100$ ). Conclusion This study demonstrates that exposure to toluene at an abuse concentration during adolescence results in specific impairments in aspects of instrumental learning, without altering motor function and spatial learning in late adolescence/early adulthood. Our data imply that persistent alterations in reward processing may occur following adolescent inhalant misuse.
\end{abstract}

Keywords: Inhalant Abuse, Cognition, Operant Self-administration, Sucrose 


\section{Introduction}

Inhalation of volatile organic solvents, such as toluene, is a significant public health concern particularly within adolescent populations. Inhalants are commonly the initial "drug" experienced as they are cheap, legal and readily accessible providing a rapid intoxicating effect (Lubman et al. 2008). Notably, adolescent inhalant abuse is a predictor of substance abuse disorders later in life, and has a significant co-morbidity with neuropsychiatric disorders, the likelihood of which is associated with age of abuse onset (Wu et al. 2004). Furthermore, adolescence is an integral maturational period in which dynamic reorganisation in white and grey matter structures occurs, particularly within regions associated with cognitive and executive functions, such as the prefrontal cortex (PFC) (Spear 2000). Consequently, the negative implications of adolescent inhalant abuse are thought to relate to the vulnerability of the maturing brain during this period (Lubman et al. 2007).

Chronic inhalant abuse results in significant neuropathological abnormalities, with human imaging studies reporting both white (Yamanouchi et al. 1995, Rosenberg et al. 2002), and grey matter lesions (Aydin et al. 2009). These abnormalities are correlated with neurological and cognitive deficits, such as ataxia, tremors, learning and memory deficits, as well as impaired visuospatial, verbal and executive functions (Rosenberg et al. 2002, Aydin et al. 2009). The severity of these motor and cognitive deficits is associated with both the duration of abuse and the severity of neuropathological abnormalities (Yucel et al. 2008). While some degree of recovery may occur with abstinence in humans, deficits in learning and memory and executive function often persist (Cairney et al. 2005, Dingwall et al. 2011), even following protracted abstinence (Cairney et al. 2013). Therefore, neural circuits mediating cognitive and executive functions may be susceptible to persistent inhalant-induced impairments.

The volatile organic solvent toluene is found in many household products that are commonly abused. Toluene has high potential for abuse as it can elicit a conditioned place preference in rodents (Funada et al. 2002) and is self-administered in mice (Blokhina et al. 
2004) and non-human primates (Weiss et al. 1979). Similar to other drugs of abuse, toluene's ability to regulate signalling within the mesocorticolimbic dopaminergic (DA) system is thought to underlie its acute hedonic and reinforcing properties (Lubman et al. 2008, Beckley et al. 2013). Toluene also induces neurobiological alterations within the medial PFC (mPFC) (Williams et al. 2005), and animal studies have demonstrated that toluene exposure can result in cognitive impairments in tasks such as novel object recognition (Lin et al. 2010, Huerta-Rivas et al. 2012), inhibitory avoidance (Huerta-Rivas et al. 2012), spatial learning (von Euler et al. 1993) and instrumental learning (Bowen and McDonald 2009). Importantly, the negative implications of adolescent toluene inhalation remain relatively unknown. Few animal studies have investigated the long-term effects of chronic adolescent toluene exposure upon locomotor activity (Batis et al. 2010), cognitive function (Lin et al. 2010, Huerta-Rivas et al. 2012), or exposure to concentrations that model human abuse settings (> 5,000 ppm) (Bowen and McDonald 2009). This is further impacted by the fact that adolescents display differential sensitivity to inhalants compared to adults in animal models (O'Leary-Moore et al. 2009 Batis et al. 2010).

The present study therefore aimed to investigate the impact of adolescent chronic intermittent toluene (CIT) exposure at an abuse concentration $(10,000 \mathrm{ppm})$ upon specific aspects of motor, spatial and reward-based learning in late adolescence and adulthood. Following a period of adolescent air (controls) or CIT exposure, separate cohorts of rats were tested in the rotarod, morris water maze, or operant self-administration paradigms to investigate discrete, as opposed to general, cognitive processes. We hypothesised that adolescent CIT exposure would impair specific learning and memory processes due to the maturational vulnerability of neural circuitry during this period. 


\section{Materials and Methods}

Animals

Adolescent male Wistar rats (PN 24; Air-exposed, $\mathrm{n}=39$; CIT-exposed, $\mathrm{n}=41$ ) were obtained from the Animal Resources Centre (Perth, Australia). In rats, adolescence ranges from weaning at PN 21 to adulthood at PN 60 (Spear 2000). Rats were pair housed, maintained on a $12 \mathrm{hr}$ light/dark cycle (lights on at 7:00) and given access to food and water ad libitum. Rats were acclimatised for 3 days prior to any experimental manipulation. All experiments were performed in accordance with the Prevention of Cruelty to Animals Act, 1986 under the guidelines of the National Health and Medical Research Council Code of Practice for the Care and Use of Animals for Experimental Purposes in Australia.

\section{Toluene inhalation exposure}

Exposure to toluene inhalation was conducted as previously described (Duncan et al. 2012). Briefly, rats were acclimatised to the laboratory at least $1 \mathrm{hr}$ prior to exposure to toluene or air during which time their body weights were recorded. Exposure to vapourised toluene was conducted in specialised chambers $(4.76 \mathrm{~L} ; 17.6 \times 16.5 \times 16.4 \mathrm{~cm})$ connected to a toluene vapour system whereby air was pumped through liquid toluene (1.08389, purity 99.8\%, Merck, Vic, Australia) in a gas wash-bottle to produce toluene vapour. Flow meters allowed the regulation of the desired concentration of toluene vapour in the exposure chambers and the concentration verified using a previously calibrated inline gas chromatography system (Shimadzu Corporation, Kyoto, Japan). A minimum of 3 readings were taken per session with deviations greater than $100 \mathrm{ppm}$ of the desired toluene concentration being corrected. Chambers of similar design but exposed to room air only were utilised for control animals ( 0 ppm exposure).

Three separate experimental cohorts were assessed (Rotarod, Morris water maze, and operant self-administration of sucrose) and behavioural testing was conducted such that each cohort was tested specifically in a single behavioural paradigm. CIT-exposed rats in the Rotarod cohort were also subjected to assessment of locomotor activity during acute recovery following the initial and final exposure to toluene. Within each experimental 
cohort rats were randomly assigned to inhale either air or toluene (10,000 ppm) for $1 \mathrm{hr}$ per day, 3 days per week (Monday, Wednesday, Friday), for 4 weeks. Thus, the 4 week exposure period took animals from early (PN 27) to late adolescence (PN 52). The chronic intermittent exposure paradigm employed was designed to mirror the human pattern of toluene abuse at an equivalent abuse concentration (Lubman et al. 2008) where the sensitivity to toluene is approximately equivalent in both species (Benignus et al. 2007). After $1 \mathrm{hr}$ of exposure, rats were placed back into their home cages and semi isolated from other rats for at least $1 \mathrm{hr}$ to avoid the possible confounds of olfactory stimulation by toluene scent on the fur. All chambers were briefly cleaned with $70 \%$ ethanol between sessions. Exposures were conducted at room temperature $\left(\sim 21^{\circ} \mathrm{C}\right)$ under normal lighting and each rat was exposed at approximately the same time each day $(\sim 9: 00-11: 00$ or $2-4$ hrs into the light cycle).

\section{Locomotor Recovery and Rotarod}

To assess recovery from the acute locomotor effects of toluene inhalation, CITexposed rats $(n=10)$ were placed in locomotor cells $\left(\operatorname{TruScan}^{\mathrm{TM}}\right.$ Photobeams; Coulbourn Instruments, Allentown, PA, USA) immediately following the initial and final exposure and horizontal (HP) and vertical plane (VP) activity recorded for 40 mins as previously described (Duncan et al. 2013). Motor learning and co-ordination following adolescent CIT exposure was then assessed. Both air- $(n=7)$ and CIT-exposed rats $(n=10)$ were tested on the Rotarod 6 days after the last exposure (PN 58). The Rotarod apparatus (Ugo Basile, Italy) consisted of a tactile rod $6 \mathrm{~cm}$ in diameter, separated via partitions into four segments $8.7 \mathrm{~cm}$ wide. Two training sessions 15 mins apart, one at a fixed speed of $4 \mathrm{rpm}$ and one accelerating from $4 \mathrm{rpm}$ to $24 \mathrm{rpm}$, were undertaken whereby each rat had to remain on the Rotarod for 2 mins. Following a $4 \mathrm{hr}$ retention period rats were subjected to three $5 \mathrm{~min}$ tests 15 mins apart on an accelerating rod ( $4 \mathrm{rpm}$ to $24 \mathrm{rpm}$ ) and the latency to fall was recorded. Motor co-ordination was quantified as increased time spent on the Rotarod prior to falling.

Morris water maze 
Spatial learning was assessed in the Morris water maze following 4 weeks of air (n $=22)$ or CIT exposure $(\mathrm{n}=23)$. The water maze $(180 \mathrm{~cm}$ diameter x $62 \mathrm{~cm}$ depth $)$ was filled with $42 \mathrm{~cm}$ of water $\left(25^{\circ} \mathrm{C}\right)$ and divided in notional quadrants with distinct visual cues positioned at the North, South, East, and West points of the pool to allow spatial reference learning to occur (Featherby et al. 2008). Training consisted of four 2 min trials per day with 30 min intervals between each trial, which commenced 3 days following the final exposure day. Rats were placed on the edge of the maze facing the wall in one of the four quadrants (different each day) and allowed to locate the submerged platform or, failing this, they were then guided to its location. Rats were allowed to remain on the platform for 30 secs and then removed from the maze. Throughout acquisition (5 days, PN 55 - 59), the platform was positioned within the South East quadrant (Fig. 3a). To assess reversal learning in this paradigm, the platform location was positioned in the North West quadrant on day 6 and day 7 (PN $60-61$ ). On day 8, the platform location was relocated to the North East quadrant and remained in this location on day 9 (PN $62-63$ ). Following reversal learning, retention of spatial memory was assessed in a single 1 min probe test on day 10 (PN 64). In this trial, the platform was removed from the pool and the time spent within each quadrant assessed. For all experimentation, tracking data was obtained with EthoVision 3.0 (Noldus Information Technology, Sterling, VA).

\section{Operant self-administration of sucrose}

Three days after the 4 week exposure period, all rats (Air $\mathrm{n}=10$; CIT $\mathrm{n}=10)$ were trained to respond for sucrose $(5 \% \mathrm{w} / \mathrm{v})$ in standard sound-attenuated operant chambers (Med Associates, VT, USA), essentially as previously described (Jupp et al. 2011). Briefly, operant chambers were equipped with 2 retractable levers on either side of the chamber adjacent to a fluid receptacle. Availability of sucrose was conditioned with the presence of a discriminative olfactory cue (vanilla essence placed directly underneath the active lever), and fulfilment of a fixed ratio (FR) requirement resulted in sucrose delivery paired with 1 sec illumination of a cue light (conditioned stimulus (CS)) located above the active lever. 
Both active and inactive lever responses within each session were recorded with each delivery consisting of $100 \mu \mathrm{l}$ of either sucrose or water respectively. Rats were trained to respond for sucrose in a single overnight session (14 hrs) on an FR2 schedule of reinforcement followed by daily $20 \mathrm{~min}$ operant sessions on an FR3 schedule of reinforcement, 5 days per week.

Acquisition consisted of 10 operant sessions (PN 57 - 70) such that lever discrimination and stable responding was established ( $>30$ active lever presses and $<15 \%$ variability over last 3 sessions) (Fig. 3a). Failure to reach these criteria resulted in exclusion from data analysis. Two CIT-exposed rats were excluded, one due to illness throughout the self-administration paradigm, while the other failed to reach the acquisition criteria (CIT $n=$ 8). Following acquisition, rats were tested under a progressive ratio (PR) schedule of reinforcement in two 90 min sessions as previously described (Jupp et al. 2011), where the response requirement increased by 1 upon each reward delivery. PR sessions were interspersed with a single FR3 session (ie over a 3 day period; PN $71-73$ ). Breakpoint was defined as the maximum rewards received, which was equal to the maximum PR requirement fulfilled. Following the second PR session, reacquisition of lever responding occurred over 2 days in 20 min FR3 sessions followed by instrumental extinction in daily 20 min sessions whereby the discriminative cue was removed from the chambers, and responding on either lever had no programmed consequences. Extinction continued for 10 consecutive days (PN $78-87)$ to allow rats to reach extinction criteria $(<10$ active lever presses or $<15 \%$ of the last FR3 session over the final 3 days of extinction). The day following extinction, cue-induced reinstatement of sucrose-seeking was assessed in a single 20 min session (PN 88) whereby lever responding was precipitated by the reintroduction of the discriminative cue under the active lever, and contingent CS presentation upon each FR3 response. A single sucrose delivery $(<100 \mu \mathrm{l})$ was received upon the first FR3 response on the active lever, with no further deliveries upon subsequent responding. A single air-exposed rat was excluded due to technical failure of the operant chamber $(n=9)$. After the single reinstatement test, reacquisition of active lever responding for sucrose 
occurred over 2 days in 20 min FR3 sessions. Following reacquisition, reversal of lever locations was employed to assess reversal learning (PN 92 - 100). Reversal sessions did not differ from former FR3 sessions, except the lever locations were reversed and the discriminative cue was positioned under the newly located active lever. Once responding was reacquired to pre-reversal levels (5 days), the levers were again reversed back to their original locations to assess performance upon a second reversal of lever locations.

\section{Statistical Analyses}

Statistical analyses were performed using IBM SPSS Statistics 20 (IBM, USA), and SigmaStat 3.5 (Jandel, San Jose, CA, USA) and all graphs were produced with GraphPad Prism 6 (GraphPad Software, San Diego, CA, USA). Body weights throughout exposure were analysed using two-way repeated measures Analysis of Variance (RM ANOVA) with treatment and time as factors. Time course of locomotor recovery following exposure in CIT-exposed rats was analysed with a two-way RM ANOVA with exposure day and time as factors; paired comparisons were employed. Rotarod data was analysed using a two-way RM ANOVA with treatment and trial as factors. Water maze data were analysed with twoway RM ANOVAs with treatment and day as factors for acquisition, and reversal sessions (each reversal session analysed individually in comparison to pre-reversal day) and treatment and quadrant as factors for retention trial data; paired comparisons were employed. For all operant self-administration studies, two-way RM ANOVAs were used to analyse acquisition (treatment $\mathrm{x}$ session), lever discrimination (treatment $\mathrm{x}$ lever), total lever responses (treatment $\mathrm{x}$ lever) and time courses (treatment $\mathrm{x}$ time) for reversal data and total lever responses (treatment $\mathrm{x}$ session) and time courses (treatment $\mathrm{x}$ time) of cue-induced reinstatement data. Holm-Sidak paired comparisons were utilised for all post hoc comparisons. Unpaired $t$ tests were employed to analyse the difference between groups in body weights upon cessation of experimentation, total swim distance in the retention trial of water maze experiments, as well as lever responses and breakpoints in PR sessions. All data are expressed as mean \pm SEM, or mean of means \pm SEM (water maze acquisition and reversal data). Significance was accepted as $p<0.05$. 


\section{Results}

Effect of adolescent CIT exposure on body weight and recovery of motor function

Throughout the 4 week exposure period toluene significantly attenuated weight gain of CIT- compared to air-exposed rats in all cohorts assessed. Analysis of grouped body weights throughout the exposure period revealed a main effect of treatment $\left(F_{(1,77)}=31.94\right.$, $p<0.001)$ and time $\left(\mathrm{F}_{(2,154)}=10364.40, p<0.001\right)$, plus a significant treatment $\mathrm{x}$ time interaction $\left(\mathrm{F}_{(2,154)}=125.62, p<0.001\right.$, Fig. 1a). Post hoc analysis revealed that body weights were reduced in CIT-exposed rats from the $6^{\text {th }}$ exposure day $(p<0.001)$. Body weights remained significantly reduced in CIT-exposed rats at the cessation of experimentation in both the Rotarod (Air; $352.1 \pm 8.6$ g; CIT $313.9 \pm 9.4 \mathrm{~g}, p<0.01$; PN 58 ) and Morris water maze cohorts (Air; $384.8 \pm 4.1 \mathrm{~g}$; CIT $333.9 \pm 4.1 \mathrm{~g}, p<0.001 \mathrm{PN}$ 64). While there was a trend towards decreased body weight in CIT- compared to airexposed rats upon cessation of experimentation in the sucrose operant cohort, this did not reach statistical significance (Air 568.6 $\pm 21.3 \mathrm{~g}$; CIT $518.6 \pm 13.9 \mathrm{~g}, p=0.08$; PN 100).

Analysis of HP activity during recovery following the initial (PN 27) and final toluene exposure (PN 52) revealed a main effect of exposure $\left(\mathrm{F}_{(1,18)}=32.59, p<0.001\right)$ and time $\left(\mathrm{F}_{(9,162)}=10.90, p<0.001\right)$ as well as an exposure $\mathrm{x}$ time interaction $\left(\mathrm{F}_{(9,162)}=3.695, p\right.$ $<0.001$, Fig. 1b). Post hoc analysis revealed significantly increased HP activity from $16-$ 32 mins, and at 40 mins recovery $(p<0.01)$ on the final compared to the initial exposure suggesting enhanced recovery from the hypolocomotor effects of toluene by the end of the exposure period. No differences in VP activity were observed between the initial and final exposure (data not shown).

Adolescent CIT exposure does not impair motor learning or co-ordination

Analysis of Rotarod performance 6 days following the final exposure day (PN 58) revealed a main effect of trial $\left(\mathrm{F}_{(2,30)}=15.39, p<0.001\right)$ but no main effect of treatment $(\mathrm{F}$ $<1)$ or a factors interaction $(\mathrm{F}=1.12$, Fig. 2$)$. Subsequent post hoc analysis revealed that both air- and CIT-exposed rats displayed significantly increased time on the Rotarod across 
the 3 trials $(p<0.05$, Fig. 2). These results demonstrate that adolescent CIT exposure did not affect motor learning or co-ordination as measured by Rotarod performance.

Spatial learning and spatial reversal learning remains intact following adolescent CIT exposure

Analysis of the latency to discover the submerged platform within the acquisition phase of the Morris water maze experiment (PN 55 - 59; Fig. 3a) revealed a significant main effect of day $\left(\mathrm{F}_{(4,172)}=80.30, p<0.001\right)$, with no main effect of treatment or a factors interaction $(F<1)$, suggesting that both groups similarly acquired the task (Fig. 3b). Following acquisition, spatial reversal learning was tested over the next 4 days (PN $60-63$ ) whereby the platform location was relocated twice ( 2 days per location). Analysis of latencies to find the submerged platform on the pre-reversal (day 5) and initial reversal day (R1, day 6) revealed a main effect of day $\left(\mathrm{F}_{(1,43)}=14.66, p<0.001\right)$ with no main effect of treatment or a factors interaction $(\mathrm{F}<1$, Fig. 3c left panel). The second reversal (R2) revealed no main effects or a factors interaction $(\mathrm{F}<1$, Fig. $3 \mathrm{c}$ right panel). Retention of spatial memory was then assessed in a single 1 min probe test (day 10, PN 64), whereby the submerged platform was removed and the time spent within each quadrant recorded. Analysis of the probe test data revealed a main effect of quadrant $\left(\mathrm{F}_{(3,129)}=37.32, p<\right.$ 0.001 ) with no main effect of treatment or a factors interaction ( $\mathrm{F}<1$, Fig. 3d). Post hoc analysis revealed significantly greater time spent in the North East quadrant where the platform was located on the previous day $(p<0.001)$ and significantly less time spent in the South West quadrant where the platform was never located $(p<0.001)$, with no differences between treatment groups (Fig. 3d). Total distance travelled during the probe test did not differ between groups (Fig. 3e). These results suggest that the acquisition, reversal and retention of a spatial learning task were not affected following adolescent CIT exposure under the current paradigm conditions.

Acquisition but not expression of instrumental responding for sucrose is impaired following adolescent CIT exposure 
For sucrose operant self-administration experiments (Fig. 4a) analysis of the initial acquisition of active lever responding for sucrose (operant sessions $1-3$, PN 57 - 59; Fig. $4 b)$, revealed a main effect of session $\left(\mathrm{F}_{(2,32)}=7.15, p<0.01\right)$, with no main effect of treatment $(\mathrm{F}=2.04)$ or a factors interaction $(\mathrm{F}=2.44)$. Post hoc analysis revealed a significant increase of active lever responding across the first 3 operant sessions in air- $(p<$ 0.001 ) but not CIT-exposed rats $(p=0.39$, Fig. 4b). However, analysis of lever discrimination on the final acquisition day (operant session 10, PN 70) revealed a main effect of lever $\left(\mathrm{F}_{(1,16)}=37.16, \mathrm{p}<0.001\right)$, with no main effect of treatment or a factors interaction $(F<1)$, suggesting that once acquired, there was no significant difference in stable responding for sucrose between groups (Fig. 4c).

Motivation to acquire sucrose was not affected following adolescent CIT exposure

Following acquisition, we employed a PR schedule of reinforcement to test motivation to acquire a sucrose reward in CIT-exposed rats. Analysis of mean active lever responding (Fig. 4d) and breakpoints (Fig. 4e) revealed no significant differences between groups (air or CIT, PN $71-73$ ). These results suggest that the motivation to obtain and consume sucrose was not affected following adolescent CIT exposure.

\section{Extinction and cue-induced reinstatement}

Analysis of active lever responding throughout extinction training (PN $78-87$ ) revealed a gradual decrease in active lever responding across days as evidenced by a main effect of day $\left(\mathrm{F}_{(9,144)}=65.23, p<0.001\right)$, with no main effect of treatment or a factors interaction $(\mathrm{F}<1$; data not shown). Once extinction criteria were met, all rats were subjected to cue-induced reinstatement to assess the effect of adolescent CIT exposure upon reward-seeking behaviour (PN 88). Analysis of total active lever responses upon cueinduced reinstatement of sucrose-seeking (Fig. 5a), revealed a main effect of session $\left(F_{(1,15)}\right.$ $=39.87, p<0.001)$, as both treatment groups displayed a significant increase of active lever responding upon presentation of cues previously associated with reward delivery, yet there was no main effect of treatment $(F<1)$ or a factors interaction $(F=1.69)$. However, analysis of the time course of active lever responding within the reinstatement session (Fig. 
$5 b)$, revealed a main effect of time $\left(\mathrm{F}_{(3,45)}=21.03, p<0.001\right)$ and a treatment $\mathrm{x}$ time interaction $\left(\mathrm{F}_{(3,45)}=3.03, p<0.05\right)$. Subsequent post hoc analysis revealed significantly lower active lever responding within the first 5 mins of the reinstatement session in CITcompared to air-exposed rats $(p<0.01$, Fig. 5b). Furthermore, CIT-exposed rats displayed a significant increase in the latency to fulfil an initial FR3 response ( $p<0.05$, Fig. 5 c).

Operant reversal learning is impaired following adolescent CIT exposure

Operant reversal learning was assessed following reacquisition of active lever responding after cue-induced reinstatement ( 2 days). Analysis of total lever responses in the initial reversal session (R1, PN 92; Fig. 6a, left panel) revealed no main effects or a factors interaction $(\mathrm{F}<1)$. However, analysis of the time course of active lever responses within $\mathrm{R} 1$ (Fig. 6b) revealed a main effect of time $\left(\mathrm{F}_{(3,48)}=7.486, p<0.001\right)$ and a treatment $\mathrm{x}$ time interaction $\left(\mathrm{F}_{(3,48)}=3.71, p<0.05\right)$. Subsequent post hoc analysis revealed that air-exposed rats significantly increased active lever responding throughout the session $(p<0.05)$, while CIT-exposed rats displayed no significant differences in active lever responding throughout this session (Fig. 6b). Analysis of total lever responses on the second reversal (R2, PN 98; Fig. 6a, right panel) revealed a main effect of lever $\left(\mathrm{F}_{(1,16)}=44.54, p<0.001\right)$ but no main effect of treatment or a factors interaction $(\mathrm{F}<1)$. No differences were observed in the time course of active lever responding between groups within R2 (data not shown). 


\section{Discussion}

In this study, we investigated the impact of chronic adolescent toluene inhalation at a concentration relevant to human abuse settings (10,000 ppm) (Bowen et al. 2006) upon subsequent motor and cognitive function in late adolescence and adulthood. Adolescent CIT exposure resulted in an attenuation of weight gain within the exposure period, which appeared to recover by around 7 weeks after exposure ceased (PN 100). Enhanced recovery from the acute hypolocomotor effects of toluene inhalation were also observed as CITexposed rats displayed a more rapid onset of increased HP activity during recovery following the final compared to the initial exposure as previously demonstrated in our model following a shorter duration of exposure (Duncan et al. 2013). Consistent with this, we have a previously demonstrated tolerance to the acute ataxic effects of adolescent CIT exposure in this model (Duncan et al. 2013). Rotarod performance in late adolescence revealed that both motor co-ordination and motor learning were not affected following adolescent CIT exposure. Furthermore, the acquisition, reversal and retention of spatial learning in the Morris water maze did not differ between air- and CIT-exposed rats. Together, these results suggest that both motor function and spatial learning are largely intact following adolescent CIT exposure in this model. However, operant responding for sucrose in adulthood revealed impairments in specific aspects of instrumental learning following adolescent CIT exposure. Thus, although the integrity of neural circuits was not directly assessed in this study, our results suggest that some circuits appear more sensitive than others to modulation following adolescent CIT exposure.

\section{Motor co-ordination and spatial learning are intact following adolescent CIT exposure}

Here we show that adolescent CIT exposure does not subsequently impair motor co-ordination or motor learning as evidenced by Rotarod performance in late adolescence. This is in line with studies assessing the effects of chronic exposure to low concentrations of toluene (< $1500 \mathrm{ppm}$ ) (Ladefoged et al. 1991, Beasley et al. 2012). Interestingly, adolescent CIT exposure did not impair acquisition, reversal or retention of spatial learning in the Morris water maze task, indicating that spatial learning and related memory processes 
are not impaired under our conditions. Thus, these results suggest a lack of persistent dysfunction within neural circuits involved in spatial learning, such as the hippocampus, following adolescent CIT exposure (Morris et al. 1986). In contrast, chronic low concentrations of toluene inhalation ( $80 \mathrm{ppm}, 6 \mathrm{hrs} /$ day, 5 days/week for 4 weeks) impaired Morris water maze performance (von Euler et al. 1993), which may reflect hippocampal neurotoxicity (Seo et al. 2010). However, our study is line with Ladefoged and colleagues (1991), who demonstrated that daily toluene inhalation (500 and $1500 \mathrm{ppm}$ ), for up to 6 months did not result in impaired acquisition or retention of spatial memory in the Morris water maze task. Acute toluene inhalation $(5,000 \mathrm{ppm}$ for $30 \mathrm{mins})$ alters hippocampal output in vivo, yet only impairs water maze performance when exposure occurs prior to each training session, suggesting transient effects upon hippocampal-dependent learning (Gmaz et al. 2012a, Gmaz et al. 2012b).

Discrepancies between previous studies and the present findings likely arise from the various exposure paradigms employed; we have previously demonstrated that the duration of the exposure period (4 compared to 8 weeks) and consequently the number of exposures (12 compared to 24 exposures) alters both the behavioural and neuropathological consequences of toluene inhalation (Duncan et al. 2012). Indeed, duration of abuse history appears to relate to the neuropathological (Yamanouchi et al. 1995, Aydin et al. 2002) and cognitive abnormalities (Cairney et al. 2005) observed in human inhalant abusers. Thus, the exposure paradigm we employed does not appear to result in generalised deficits in learning and memory processes, arguing against overt neurotoxicity or dysfunction in regions required for motor learning and co-ordination or spatial learning.

\section{Adolescent CIT exposure results in specific impairments in aspects of instrumental learning}

The implications of chronic adolescent toluene exposure on subsequent instrumental learning remains relatively unknown, although studies have assessed the effects of both acute and chronic toluene inhalation on reinforced operant responding in adult animals (Bowen et al. 2006). Adolescent CIT exposure delayed the initial acquisition of instrumental responding for sucrose. However, after acquisition no differences were 
observed between air- or CIT-exposed rats suggesting that expression of instrumental responding was intact. In line with these findings, daily toluene inhalation (1000 ppm) for 13 weeks, resulted in delayed acquisition of instrumental responding in a simple signal detection task, where no deficits were observed once the task was acquired (Beasley et al. 2012). Furthermore, Bowen and McDonald, 2009, demonstrated that daily toluene inhalation (3,600 and 6,000 ppm for 40 days) following initial operant training, impaired responding for sweetened condensed milk in an instrumental "wait for reward" task, where performance was impaired throughout the exposure period (testing $22-23 \mathrm{hrs}$ following daily exposures), as well as up to 40 days of recovery in mice exposed to $6,000 \mathrm{ppm}$. The latter study suggests that following training, inhalation of toluene may persistently impair retention/retrieval of a previously learnt instrumental task, although potential motor deficits or amotivation may also account for these impairments (Bowen and McDonald 2009). In our study, motor deficits were not observed and responding on PR schedules did not differ between air- and CIT-exposed rats suggesting that the motivation to acquire sucrose was similar between groups and therefore should not confound initial responding in CITexposed rats.

Although reversal learning deficits were not observed in the Morris water maze, reversal of lever locations in operant experiments revealed that adolescent CIT exposure modestly impaired operant reversal learning within the initial session (R1). This was evident as air- but not CIT-exposed rats significantly increased responding on the newly located active lever throughout this session. This impairment was observed 40 days following the final toluene exposure yet was not evident in subsequent reacquisition sessions or a second reversal session (R2). Badanich et al., 2011, demonstrated that posttraining chronic intermittent ethanol vapour inhalation, resulted in a transient deficit in reversal learning present at $72 \mathrm{hrs}$ but not 14 days withdrawal, with no effects on a mPFCmediated set-shifting task, suggestive of preferential vulnerability of the orbitofrontal cortex (OFC). Reversal-specific impairments have also been observed following cocaine (Calu et al. 2007, Schoenbaum et al. 2004) and methamphetamine administration (Izquierdo et al. 
2010). However, lesions of the OFC result in perseverative errors (ie failure to inhibit responding to previously rewarded stimulus) in reversal learning tasks (Schoenbaum et al. 2002, Chudasama and Robbins 2003), which were not observed in the current study. Thus, it is unlikely that adolescent CIT exposure results in overt damage of regions implicated in reversal learning but persistent functional alterations within these regions may contribute to the initial reversal learning deficit in the present study.

While instrumental extinction was not different between groups, upon cue-induced reinstatement of sucrose-seeking, CIT exposed rats showed attenuated active lever responding early in the session. This resulted in an increased latency to achieve the initial FR3 response, and thus the initial CS presentation. As cue-induced reinstatement was precipitated by the re-introduction of the discriminative cue and contingent CS presentation, these results suggest that the excitatory effect of the discriminative cue may have been greater in air- compared to CIT-exposed rats. This may account for the potentiated responding of air- compared to CIT-exposed rats within the first 5 mins of this session. As the discriminative cue may be considered a contextual association to sucrose availability, adolescent CIT exposure may affect the acquisition of context-reward associations and/or the processing of contextual information, which may contribute to the delay in initial responding in CIT-exposed rats. Nevertheless, despite a delay in responding, CIT-exposed rats reinstated to a similar magnitude as air-exposed rats overall, arguing against overall generalised reduction in the incentive salience of the conditioned stimuli.

Adolescent CIT exposure did not alter motor or spatial learning and related memory processes, yet the specific impairments in acquisition, reversal and cue-induced reinstatement of operant responding for sucrose suggest that toluene may persistently affect the function of circuits involved in instrumental learning. Indeed, the maturational trajectory of discrete neural circuits during adolescence may attribute certain regions with a vulnerability to the effects of adolescent CIT exposure. For example, prefrontal regions continue to mature beyond the onset of early adulthood (Spear 2000, Giedd 2004), and the refinement of the mesocorticolimbic DA system, integral for instrumental learning and 
goal-directed behaviour, occurs throughout adolescence, and is particularly vulnerable to alterations during this period (Manitt et al. 2011). Consequently, toluene exposure during adolescence may affect these maturational processes resulting in persistent behavioural dysfunction in adulthood. For example a recent study demonstrated that brief toluene inhalation $(5,700 \mathrm{ppm}$ in two 10 mins exposures) in adolescent rats (PN $40-47)$ induces persistent (up to 21 days) synaptic plasticity of mesoaccumbal but not mesocortical projecting DA neurons within the VTA (Beckley et al. 2013) and that inhibition of the mPFC prior to inhalation regulated this toluene-induced plasticity. This suggests that toluene inhalation attenuates glutamatergic output from the mPFC, thereby enabling synaptic adaptation within the VTA (Beckley et al. 2013). We have also shown long-term changes to the glutamatergic system following adolescent CIT exposure presenting as altered behavioural responses to acute administration of the NMDA receptor antagonist, MK-801 (Duncan et al. 2013). While we did not directly investigate the integrity of neural circuits in this study, the behavioural deficits observed in specific aspects of operant responding for sucrose presumably reflect altered functional integrity of neural circuits mediating these behaviours.

Acquisition of instrumental responding requires functional integrity of the mPFC, which is involved in the formation, but not the expression of action-outcome (A-O) associations (Corbit and Balleine 2003, Ostlund and Balleine 2005). Thus, adolescent CIT exposure may alter the function of the $\mathrm{mPFC}$ resulting in the delayed acquisition of $\mathrm{A}-\mathrm{O}$ associations and consequently attenuate initial instrumental responding for sucrose compared to air-exposed rats. The mPFC is also required for cue elicited reward-seeking behaviours (McFarland et al. 2003, Ishikawa et al. 2008), and encoding of contextual information in operant conditioning (specifically anterior cingulate cortex) (Torregrossa et al. 2013). Thus, altered function of the mPFC in CIT-exposed rats may also contribute to the deficits observed upon cue-induced reinstatement. However, regions such as the basolateral amygdala (BLA) are also involved in cue- and context-induced reinstatement of reward-seeking as well as the acquisition of Pavlovian associations and processing of the 
incentive salience of rewards and conditioned stimuli to mediate goal-directed behaviour (Blundell et al. 2003, Lasseter et al. 2011, Balleine et al. 2003, Corbit and Balleine 2005). Thus, the behavioural deficits observed implicate several structures that may be vulnerable to adolescent CIT exposure.

\section{Limitations}

Adolescent CIT exposure attenuated weight gain within the exposure period, recovering to similar levels as air-exposed rats by 7 weeks following the exposure period (PN 100). Thus, metabolic dysfunction may have altered the incentive salience of sucrose rewards in operant self-administration experiments initially (Jarosz et al. 2008). Nevertheless, the recovery of body weight in CIT-exposed rats suggests the lack of persistent metabolic dysfunction under the present conditions. Furthermore, rats were fed ad libitum unlike many reward-based learning studies where food restriction is employed to increase the motivation to acquire food rewards (Corbit \& Balleine 2005). It must also be noted that toluene inhalation (1,000 ppm, 5 hrs/day, 5 days/week for 4 weeks), induces transient deficits in olfactory functioning associated with degenerative effects on olfactory epithelium, which recovers within $1-2$ weeks following the exposure period (Jacquot et al. 2006). Thus, adolescent CIT exposure could in theory affect olfaction, impairing the ability of CIT-exposed rats to utilise the discriminative olfactory cue to guide instrumental performance in this study. However, considering the extended duration of operant selfadministration experiments, and the lack of overall effects on responding between air- and CIT-exposed rats it appears these potential confounds are not likely to be present in the current study.

Conclusion

Adolescent CIT exposure in rats results in specific impairments in aspects of instrumental learning, without altering motor function and spatial learning in late adolescence and adulthood. These impairments were persistent, suggesting susceptibility of specific neural circuits and support the hypothesis that adolescent CIT exposure alters the 
functioning of discrete brain regions (rather than generalised "toxicity"), ultimately resulting in deficits of reward-based learning in adulthood.

1

2

3

4

5

6

7

8

9

10

11

12

13

14

15

16

17

18

19

20

21

22

23

24

25

26

27

28

29

30

31

32

33

34

35

36

37

38

39

40

41

42

43

44

45

46

47

48

49

50

51

52

53

54

55

56

57

58

59

60 
1

2

3

4

5

6

7

8

9

10

11

12

13

14

15

16

17

18

19

20

21

22

23

24

25

26

27

28

29

30

31

32

33

34

35

36

37

38

39

40

41

42

43

44

45

46

47

48

49

50

51

52

53

54

55

56

57

58

59

60

\section{Acknowledgements}

The authors would like to thank Ms. Zakia Haque for assistance with running animals through the inhalant exposure paradigm. This study was supported by The National Health and Medical Research Council of Australia of which AJL is a Principal Research Fellow (1020737), the Australian Research Council (DP 110100379) of which JRD is a Future Fellow (100100235) and the Victorian Government's Operational Infrastructure Support Scheme. 


\section{References}

Aydin K, Kircan S, Sarwar S, Okur O, Balaban E (2009) Smaller gray matter volumes in frontal and parietal cortices of solvent abusers correlate with cognitive deficits. AJNR Am J Neuroradiol 30:1922-1928.

Aydin K, Sencer S, Demir T, Ogel K, Tunaci A, Minareci O (2002) Cranial MR Findings in Chronic Toluene Abuse by Inhalation. AJNR Am J Neuroradiol 23:1173-1179.

Badanich KA, Becker HC, Woodward JJ (2011) Effects of chronic intermittent ethanol exposure on orbitofrontal and medial prefrontal cortex-dependent behaviors in mice. Behav Neurosci 125:879-891.

Balleine BW (2005) Neural bases of food-seeking: affect, arousal and reward in corticostriatolimbic circuits. Physiol Behav 86:717-730.

Balleine BW, Killcross AS, Dickinson A (2003) The effect of lesions of the basolateral amygdala on instrumental conditioning. J Neurosci 23:666-675.

Batis JC, Hannigan JH, Bowen SE (2010) Differential effects of inhaled toluene on locomotor activity in adolescent and adult rats. Pharmacol Biochem Behav 96:438448.

Beasley TE, Evansky PA, Bushnell PJ (2012) Behavioral effects of sub-acute inhalation of toluene in adult rats. Neurotoxicol Teratol 34:83-89.

Beckley JT, Evins CE, Fedarovich H, Gilstrap MJ, Woodward JJ (2013) Medial prefrontal cortex inversely regulates toluene-induced changes in markers of synaptic plasticity of mesolimbic dopamine neurons. J Neurosci 33:804-813.

Benignus VA, Boyes WK, Kenyon EM, Bushnell PJ (2007) Quantitative comparisons of the acute neurotoxicity of toluene in rats and humans. Toxicol Sci 100:146-155.

Blokhina EA, Dravolina OA, Bespalov AY, Balster RL, Zvartau EE (2004) Intravenous self-administration of abused solvents and anesthetics in mice. Eur J Pharmacol 485:211-218.

Blundell P, Hall G, Killcross S (2003) Preserved sensitivity to outcome value after lesions of the basolateral amygdala. J Neurosci 23:7702-7709. 
Bowen SE, Batis JC, Peaz-Martinez N, Cruz SL (2006) The last decade of solvent research in animal models of abuse: Mechanistic and behavioural studies. Neurotoxicol Teratol 28:636-647.

Bowen SE, McDonald P (2009) Abuse pattern of toluene exposure alters mouse behavior in a waiting-for-reward operant task. Neurotoxicol Teratol 31:18-25.

Cairney S, Maruff P, Burns CB, Currie J, Currie BJ (2005) Neurological and cognitive recovery following abstinence from petrol sniffing. Neuropsychopharmacology 30:1019-1027.

Cairney S, N OC, Dingwall KM, Maruff P, Shafiq-Antonacci R, Currie J, Currie BJ (2013) A prospective study of neurocognitive changes 15 years after chronic inhalant abuse. Addiction 108:1107-1114.

Calu DJ, Stalnaker TA, Franz TM, Singh T, Shaham Y, Schoenbaum G (2007) Withdrawal from cocaine self-administration produces long-lasting deficits in orbitofrontaldependent reversal learning in rats. Learn Mem 14:325-328.

Chudasama Y, Robbins TW (2003) Dissociable contributions of the orbitofrontal and infralimbic cortex to pavlovian autoshaping and discrimination reversal learning: further evidence for the functional heterogeneity of the rodent frontal cortex. J Neurosci 23:8771-8780.

Corbit LH, Balleine BW (2003) The role of prelimbic cortex in instrumental conditioning. Behav Brain Res 146:145-157.

Corbit LH, Balleine BW (2005) Double dissociation of basolateral and central amygdala lesions on the general and outcome-specific forms of pavlovian-instrumental transfer. J Neurosci 25:962-970.

Dingwall KM, Maruff P, Fredrickson A, Cairney S (2011) Cognitive recovery during and after treatment for volatile solvent abuse. Drug Alcohol Depend 118:180-185

Duncan JR, Dick AL, Egan G, Kolbe S, Gavrilescu M, Wright D, Lubman DI, Lawrence AJ (2012) Adolescent toluene inhalation in rats affects white matter maturation with the potential for recovery following abstinence. PloS one 7:e44790. 
Duncan JR, Gibbs SJ, Lawrence AJ (2013) Chronic intermittent toluene inhalation in adolescent rats alters behavioural responses to amphetamine and MK801. Eur Neuropsychopharmacol. In press, doi: 10.1016/j.euroneuro.2013.06.001

Featherby T, van den Buuse M, Lubman DI, Lawrence AJ (2008) Persistent downregulation of hippocampal CREB mRNA parallels a Y-maze deficit in adolescent rats following semi-chronic amphetamine administration. Br J Pharmacol 154:417-428.

Funada M, Sato M, Makino Y, Wada K (2002) Evaluation of rewarding effect of toluene by the conditioned places preference procedure in mice. Brain Res Protoc 10:47-54.

Gerasimov MR, Schiffer WK, Marstellar D, Ferrieri R, Alexoff D, Dewey SL (2002) Toluene inhalation produces regionally specfifc changes in the extracellular dopamine. Drug Alcohol Depend 65:243-251.

Giedd JN (2004) Structural magnetic resonance imaging of the adolescent brain. Ann N Y Acad Sci 1021:77-85.

Gmaz JM, Matthews BA, McKay BE (2012a) Toluene inhalation modulates dentate gyrus granule cell output in vivo. Neurotoxicol Teratol 34:403-412.

Gmaz JM, Yang L, Ahrari A, McKay BE (2012b) Binge inhalation of toluene vapor produces dissociable motor and cognitive dysfunction in water maze tasks. Behav Pharmacol 23:669-677.

Huerta-Rivas A, Lopez-Rubalcava C, Sanchez-Serrano SL, Valdez-Tapia M, Lamas M, Cruz SL (2012) Toluene impairs learning and memory, has antinociceptive effects, and modifies histone acetylation in the dentate gyrus of adolescent and adult rats. Pharmacol Biochem Behav 102:48-57.

Ishikawa A, Ambroggi F, Nicola SM, Fields HL (2008) Contributions of the amygdala and medial prefrontal cortex to incentive cue responding. Neuroscience 155:573-584.

Izquierdo A, Belcher AM, Scott L, Cazares VA, Chen J, O'Dell SJ, Malvaez M, Wu T, Marshall JF (2010) Reversal-specific learning impairments after a binge regimen of methamphetamine in rats: possible involvement of striatal dopamine. Neuropsychopharmacology 35:505-514. 
Jacquot L, Pourie G, Buron G, Monnin J, Brand G (2006) Effects of toluene inhalation exposure on olfactory functioning: behavioral and histological assessment. Toxicol Lett 165:57-65.

Jarosz PA, Fata E, Bowen SE, Jen KL, Coscina DV (2008) Effects of abuse pattern of gestational toluene exposure on metabolism, feeding and body composition. Physiol Behav 93:984-993.

Jupp B, Krivdic B, Krstew E, Lawrence AJ (2011) The orexin(1) receptor antagonist SB334867 dissociates the motivational properties of alcohol and sucrose in rats. Brain Res 1391:54-59.

Ladefoged O, Strange P, Moller A, Lam HR, Ostergaard G, Larsen JJ, Arlien-Soborg P (1991) Irreversible effects in rats of toluene (inhalation) exposure for six months. Pharmacol Toxicol 68:384-390.

Lasseter HC, Wells AM, Xie X, Fuchs RA (2011) Interaction of the basolateral amygdala and orbitofrontal cortex is critical for drug context-induced reinstatement of cocaine-seeking behavior in rats. Neuropsychopharmacology 36:711-720.

Lin BF, Ou MC, Chung SS, Pang CY, Chen HH (2010) Adolescent toluene exposure produces enduring social and cognitive deficits in mice: an animal model of solvent-induced psychosis. World J Biol Psychiatry 11:792-802.

Lubman DI, Yucel M, Hall WD (2007) Substance use and the adolescent brain: A toxic combination. J Psychopharmacol 21:792-794.

Lubman DI, Yucel M, Lawrence AJ (2008) Inhalant abuse among adolescents: neurobiological considerations. Br J Pharmacol 154:316-326.

Manitt C, Mimee A, Eng C, Pokinko M, Stroh T, Cooper HM, Kolb B, Flores C (2011) The Netrin Receptor DCC Is Required in the Pubertal Organization of Mesocortical Dopamine Circuitry. J Neurosci 31:8381-8394.

McFarland K, Lapish CC, Kalivas PW (2003) Prefrontal glutamate release into the core of the nucleus accumbens mediates cocaine-induced reinstatement of drug-seeking behavior. J Neurosci 23:3531-3537. 
Morris RG, Hagan JJ, Rawlins JN (1986) Allocentric spatial learning by hippocampectomised rats: a further test of the "spatial mapping" and "working memory" theories of hippocampal function. Q J Exp Psychol B 38:365-395.

O'Leary-Moore SK, Galloway MP, McMechan AP, Irtenkauf S, Hannigan JH, Bowen SE (2009) Neurochemical changes after acute binge toluene inhalation in adolescent and adult rats: A high-resolution magnetic resonance spectroscopy study. Neurotoxicol Teratol 31:382-389.

Ostlund SB, Balleine BW (2005) Lesions of medial prefrontal cortex disrupt the acquisition but not the expression of goal-directed learning. J Neurosci 25:7763-7770.

Rosenberg NL, Grigsby J, Dreisbach J, Busenbark D, Grigsby P (2002) Neuropsychologic impairment and MRI abnormalities associated with chronic solvent abuse. J Toxicol Clin Toxicol 40:21-34.

Schoenbaum G, Nugent SL, Saddoris MP, Setlow B (2002) Orbitofrontal lesions in rats impair reversal but not acquisition of go, no-go odor discriminations. Neuroreport 13:885-890.

Schoenbaum G, Saddoris MP, Ramus SJ, Shaham Y, Setlow B (2004) Cocaine-experienced rats exhibit learning deficits in a task sensitive to orbitofrontal cortex lesions. Eur $\mathrm{J}$ Neurosci 19:1997-2002.

Seo HS, Yang M, Song MS, Kim JS, Kim SH, Kim JC, Kim H, Shin T, Wang H, Moon C (2010) Toluene inhibits hippocampal neurogenesis in adult mice. Pharmacol Biochem Behav 94:588-594.

Spear LP (2000) The adolescent brain and age-related behavioral manifestations. Neurosci Biobehav Rev 24:417-463.

Torregrossa MM, Gordon J, Taylor JR (2013) Double dissociation between the anterior cingulate cortex and nucleus accumbens core in encoding the context versus the content of pavlovian cocaine cue extinction. J Neurosci 33:8370-8377.

von Euler G, Ogren SO, Li XM, Fuxe K, Gustafsson JA (1993) Persistent effects of subchronic toluene exposure on spatial learning and memory, dopamine-mediated 
locomotor activity and dopamine D2 agonist binding in the rat. Toxicology 77:223232.

Weiss B, Wood RW, Macys DA (1979) Behavioral toxicology of carbon disulfide and toluene. Environ Health Perspect 30:39-45.

Williams JM, Stafford D, Steketee JD (2005) Effects of repeated inhalation of toluene on ionotropic GABA A and glutamate receptor subunit levels in rat brain. Neurochem Int 46:1-10.

Wu LT, Pilowsky DJ, Schlenger WE (2004) Inhalant abuse and dependence among adolescents in the United States. J Am Acad Child Adolesc Psychiatry 43:12061214.

Yamanouchi N, Okada S-i, Kodama K, Hirai S, Hiroshi S, Murakami A, Komatsu N, Sakamot T, Sato T (1995) White Matter Changes Caused by Chronic Solvent Abuse. AJNR AM J Neuroradiol 16:1643-1649.

Yucel M, Takagi M, Walterfang M, Lubman DI (2008) Toluene misuse and long-term harms: A systematic review of the neuropsychological and neuroimaging literature. Neurosci Biobehavioural Rev 32:910-926. 


\section{Figure Captions}

Fig. 1 Adolescent CIT exposure attenuates weight gain throughout the exposure period (a) and results in enhanced recovery from the hypolocomotor effects of toluene inhalation (b). Body weights of CIT- $(n=41)$ compared to air-exposed rats $(n=39)$ were significantly reduced by the $6^{\text {th }}$ exposure day (Postnatal day (PN) 38), with decreased weights remaining at the cessation of the exposure period (PN 52) $(\mathbf{a} ; * * * p<0.001$, Air vs CIT; Two-way RM ANOVA with Holm Sidak post hoc analysis). Enhanced recovery from the acute hypolocomotor effects of adolescent CIT exposure occurred between the initial and final toluene exposure evidenced as significantly increased horizontal plane (HP) activity in the recovery period upon the final toluene exposure $\left(\mathbf{b} ; *^{* *} p<0.01, * * * p<0.001\right.$, Initial $v s$ CIT; Two-way RM ANOVA with Holm Sidak post hoc analysis). Data are mean $\pm \mathrm{SEM}$

Fig. 2 Adolescent CIT exposure has no persistent effects on motor co-ordination or motor learning as assessed in the Rotarod task. Latency to fall from Rotarod significantly increased across 3 trials for both air $(n=7)$ and CIT-exposed rats $(n=10)$ with no differences observed between groups $(\$ p<0.05, \mathrm{~T} 1 v s \mathrm{~T} 2$, T3; Two-way RM ANOVA with Holm Sidak post hoc analysis). Data are mean \pm SEM. (T1) Trial 1, (T2) Trial 2, (T3) Trial 3

Fig. 3 Adolescent CIT exposure does not impair spatial learning as measured in the Morris water maze (Timeline of Morris water maze protocol; a; Time points represented in the graphs are indicated by $\mathrm{b}, \mathrm{c}, \mathrm{d}$, and e). Average time to discover the submerged platform across training days did not differ between air- $(\mathrm{n}=22)$ and CIT-exposed rats $(\mathrm{n}=23)$, with both groups displaying a similar decrease in the latency to discover the submerged platform over repeated trials (b). Upon reversal, latency to discover the platform increased in both groups on the first (R1, left panel; main effect of day; Two-way RM ANOVA) but not second reversal (R2, right panel), with no differences observed between groups (c). In a single 1 min probe test, time spent within each quadrant did not differ between groups (d). 
Both air- and CIT-exposed rats displayed significantly increased time in the North East (NE) quadrant where the platform was located on day 9, and significantly decreased time in the South West (SW) quadrant where the platform was never located (d; \#\#\# $p<0.001$, NE $v s \mathrm{NW}, \mathrm{SE}, \mathrm{SW} ; \$ \$ \$ p 0.001, \mathrm{SW} v s \mathrm{NE}, \mathrm{NW}, \mathrm{SE}$; Two-way RM ANOVA with Holm Sidak post hoc analysis). Total distance travelled within the probe test did not differ between groups (e). Data are mean of means $\pm \operatorname{SEM}(\mathbf{b}, \mathbf{c})$, or mean $\pm \operatorname{SEM}(\mathbf{d}, \mathbf{e})$. (PN) Post natal day, (REC) Recovery, (NW) North West quadrant, (SE) South East quadrant

Fig. 4 Adolescent CIT exposure results in a delay in the initial acquisition but not expression of active lever responding for $5 \%$ sucrose with no differences in the motivation to acquire sucrose rewards between groups (Timeline of sucrose operant self-administration experiments; a; Time points represented in the graphs are indicated with b, c, d, and e). Air$(\mathrm{n}=10)$ but not CIT-exposed rats $(\mathrm{n}=8)$ significantly increased active lever responding across the first 3 operant sessions ( $\mathbf{b}$; \# $p<0.01$, Air day $3 v$ day 1,2; Two-way RM ANOVA with Holm Sidak post hoc analysis). Once responding for sucrose was acquired, lever discrimination did not differ between groups (c). No significant differences were observed in progressive ratio sessions as mean total active lever responses (d) and breakpoints (defined as maximum rewards acquired) did not differ between groups (e). Data are mean of means \pm SEM. (PN) Post natal day, (REC) Recovery, (PR) Progressive ratio, (REACQ) Reacquisition sessions, (REINST) Cue-induced reinstatement session, (R1) Initial reversal sessions, (R2) Second reversal sessions

Fig. 5 Adolescent CIT exposure results in a delay of active lever responding upon cueinduced reinstatement of sucrose-seeking. An increase in total active lever responses upon cue-induced reinstatement of sucrose-seeking (REINST) compared to the final day of extinction (EXT) was observed in both air- $(n=9)$ and CIT-exposed rats $(n=8)(\mathbf{a}$; main effect of session; Two-way RM ANOVA), with no differences between groups. However, CIT-exposed rats displayed a significant reduction in active lever responding compared to 
air-exposed rats within the first 5 mins of the reinstatement session $(\mathbf{b} ; * p<0.05$, Air $v s$ CIT; Two-way RM ANOVA with Holm Sidak post hoc analysis). This appeared to be due in part to a significant increase in the latency to the initial FR3 response in CIT-exposed rats (c; $* p<0.05$; Unpaired $t$ test). Data are mean \pm SEM

Fig. 6 Operant reversal learning is initially impaired following adolescent CIT exposure. Upon the initial operant reversal session $(\mathrm{R} 1)$ both air- $(\mathrm{n}=10)$ and CIT-exposed rats $(\mathrm{n}=$ 8) displayed a similar lack of lever discrimination with no significant differences observed between the groups (a, left panel). However, when assessing the time course of active lever responses within R1, air-exposed rats displayed a significant increase in active lever responding throughout this session (b; \# $p<0.01$, Air 5 mins vs 15 mins, 20 mins; Twoway RM ANOVA with Holm Sidak post hoc analysis), which was not observed in CITexposed rats. Upon the second operant reversal session (R2), both air- and CIT-exposed rats displayed similar active lever discrimination (a, right panel; main effect of lever; Two-way RM ANOVA). Data are mean \pm SEM 

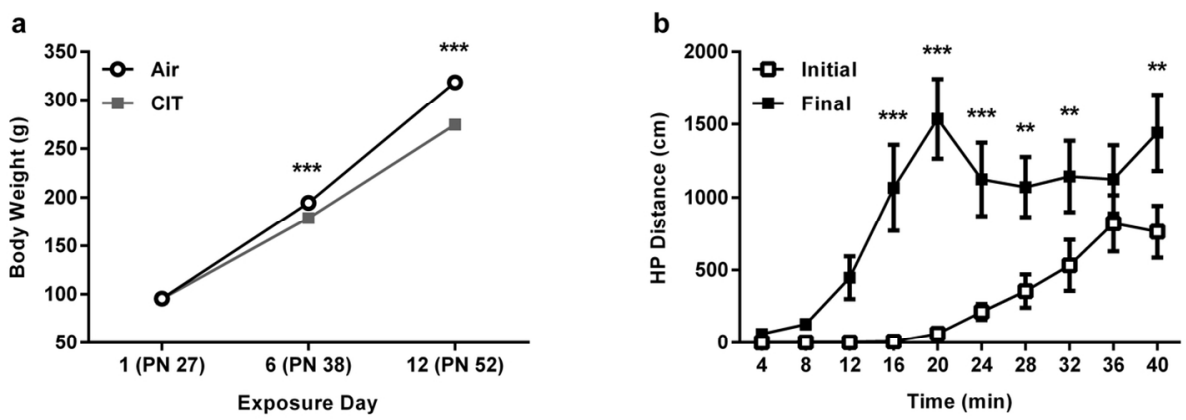

$63 \times 22 \mathrm{~mm}(600 \times 600 \mathrm{DPI})$

1

2

3

4

5

6

7

9

10

11

12

13

14

16

17

18

19

20

21

22

23

24

25

26

27

28

29

30

31

32

33

34

35

36

37

38

39

40

41

42

43

44

45

46

47

48

49

50

51

52

53

54

55

56

57

58

59

60 


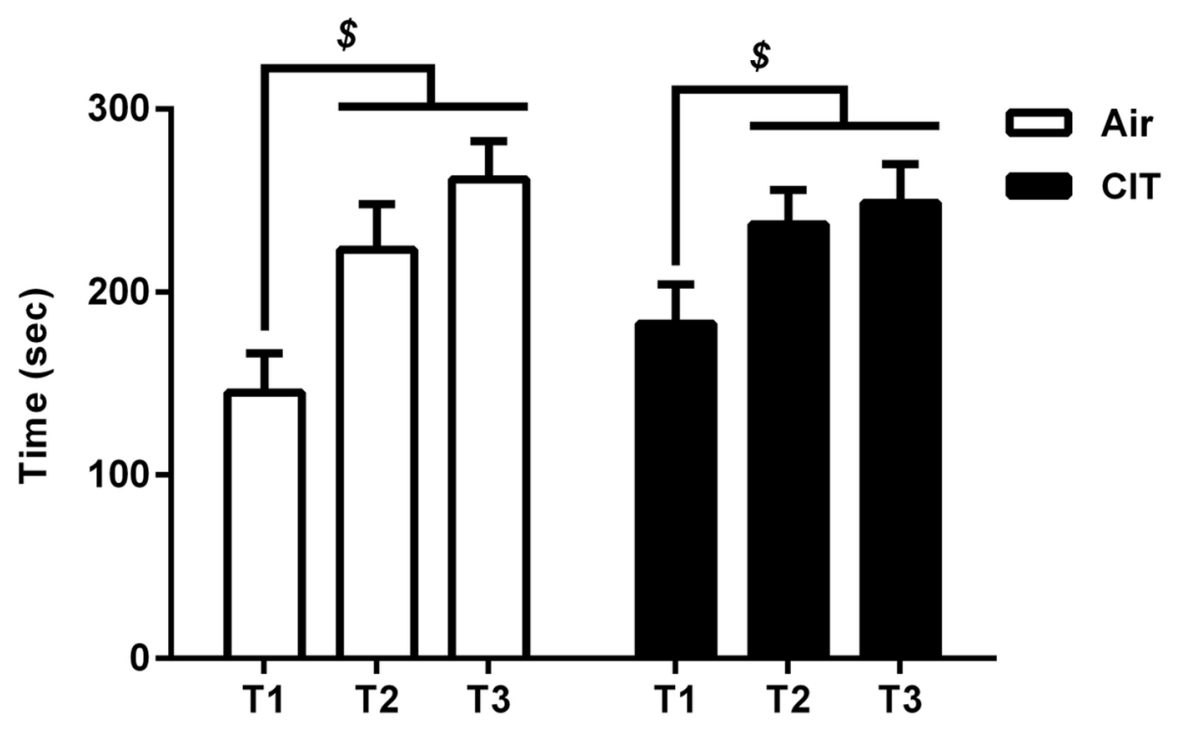

$56 \times 37 \mathrm{~mm}(600 \times 600 \mathrm{DPI})$ 
a

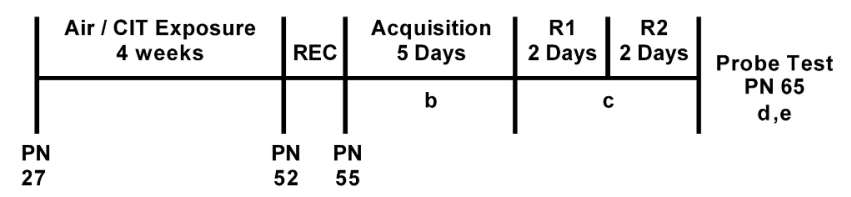

b

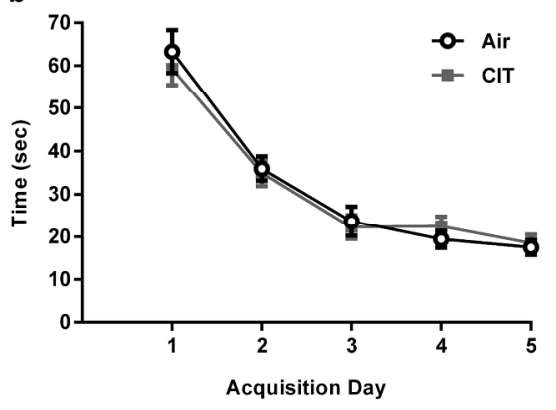

d

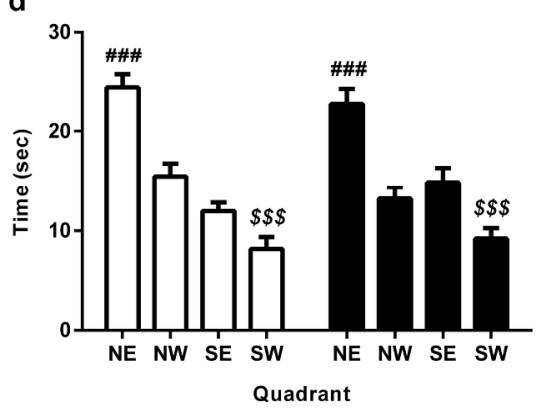

c
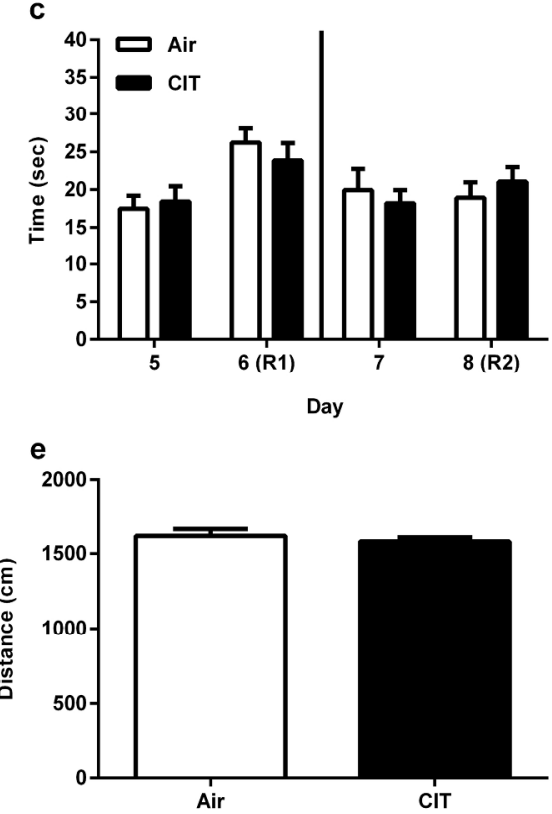
$116 \times 105 \mathrm{~mm}(600 \times 600 \mathrm{DPI})$ 
b

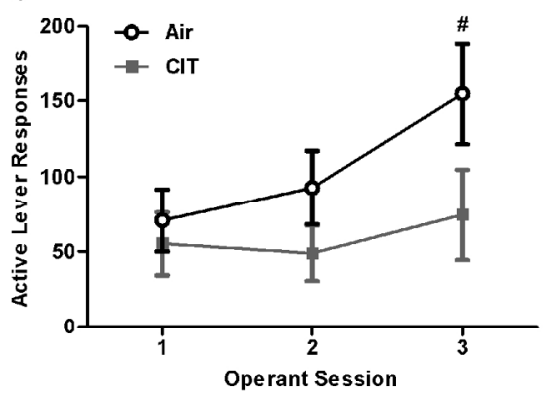

d

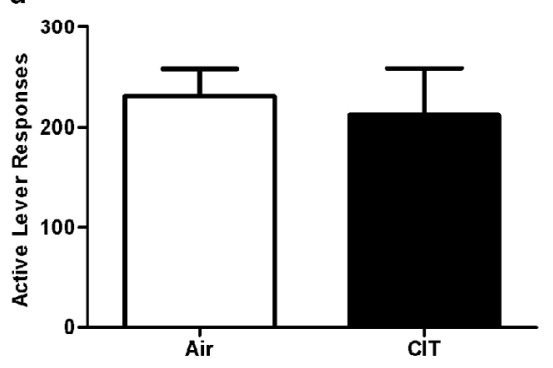

c

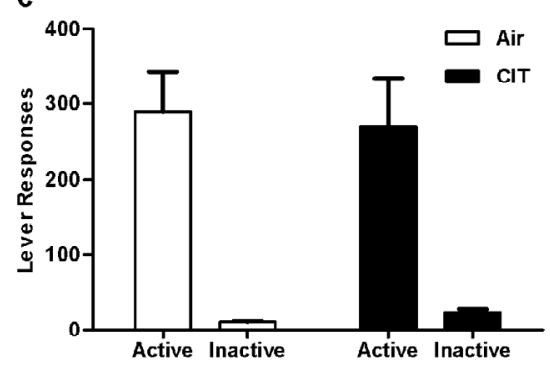

e

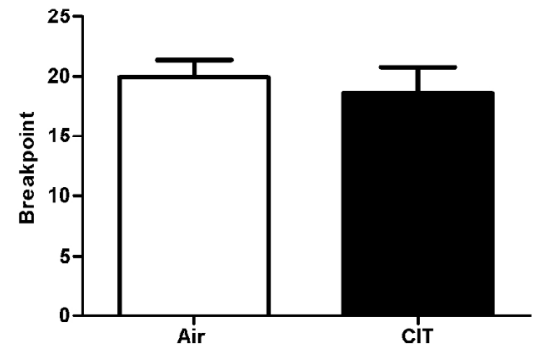

$173 \times 151 \mathrm{~mm}(300 \times 300 \mathrm{DPI})$ 
a

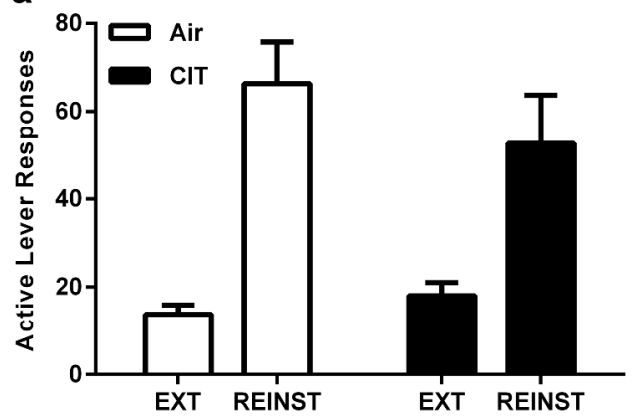

b

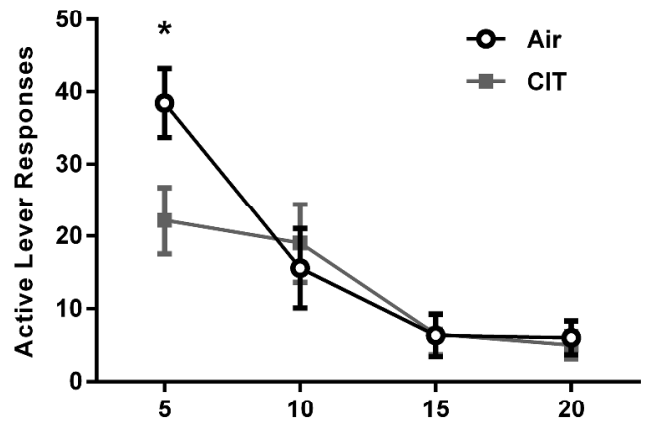

C

Time (min)

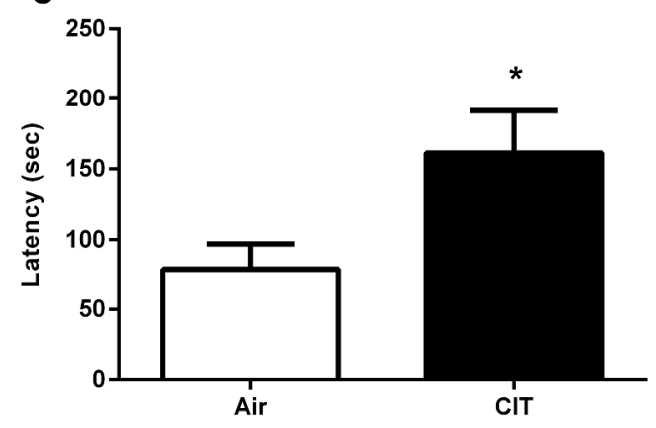

$160 \times 304 \mathrm{~mm}(600 \times 600 \mathrm{DPI})$ 

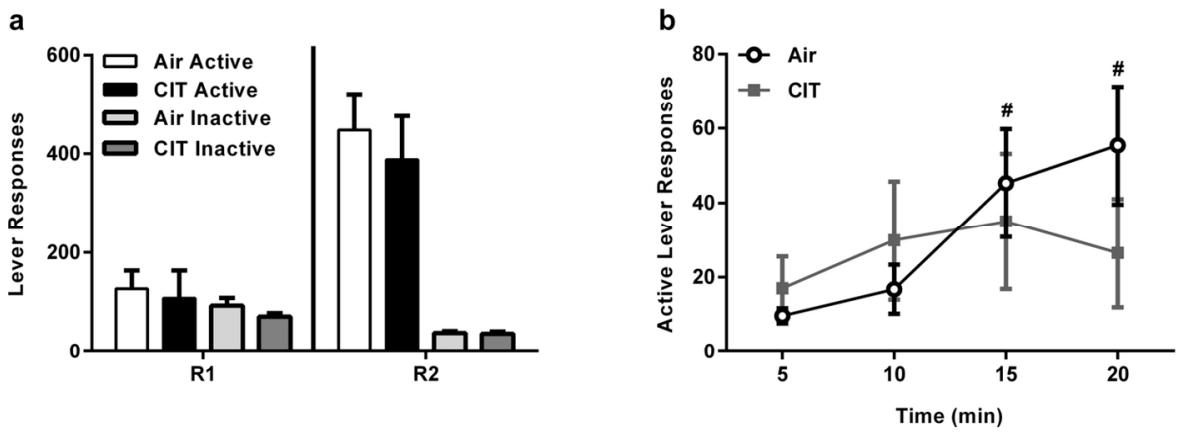

$68 \times 25 \mathrm{~mm}(600 \times 600 \mathrm{DPI})$ 


\section{Springer}

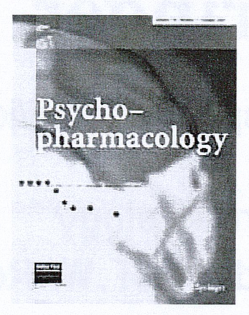

\section{Conflict of Interest Disclosure Form}

It is the policy of the Psychopharmacology to ensure balance, independence, objectivity, and scientific rigor in the Journal. All authors are expected to disclose to the readers any real or apparent conflict(s) of interest that may have a direct bearing on the subject matter of the article. This pertains to relationships with pharmaceutical companies, biomedical device manufacturers or other corporations whose products or services may be related to the subject matter of the article or who have sponsored the study.

The intent of the policy is not to prevent authors with a potential conflict of interest from publication. It is merely intended that any potential conflict should be identified openly so that the readers may form their own judgements about the article with the full disclosure of the facts. It is for the readers to determine whether the authors' outside interest may reflect a possible bias in either the exposition or the conclusions presented.

The corresponding author will complete and submit this form, along with his or her submission, to the responsible Editor on behalf of all authors listed below.

Article Title

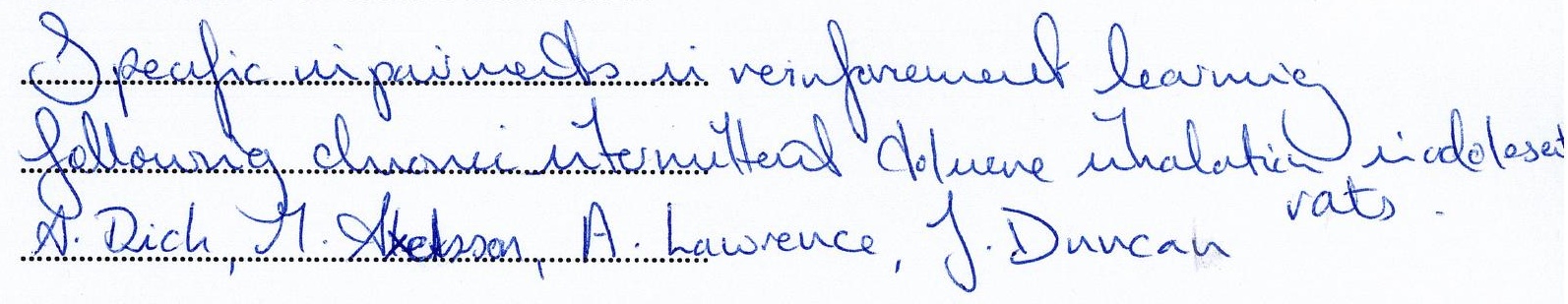

Please note that a conflict of interest statement is published with each paper.

I certify that there is no actual or potential conflict of interest in relation to this article. If any conflict exists, please define hereafter:

Conflict (if none, "None" or describe financial interest/arrangement with one or more organizations that could be perceived as a real or apparent conflict of interest in the context of the subject of this article):
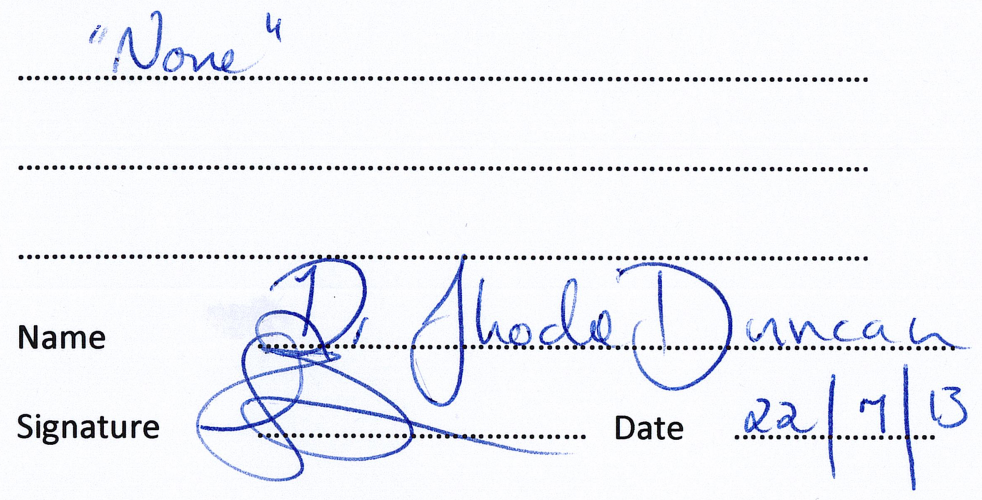

Please scan the completed and signed form and then submit it as an electronic file along with your manuscript. No manuscripts will be accepted for review without this form. 
Title: Specific impairments in instrumental learning following chronic intermittent toluene inhalation in adolescent rats

\author{
Alec L.W. Dick ${ }^{1,2}$, Martin Axelsson ${ }^{1,4}$, Andrew J. Lawrence ${ }^{1,2} \&$ Jhodie R. Duncan ${ }^{1,3}$ \\ ${ }^{1}$ Florey Institute of Neuroscience \& Mental Health; ${ }^{2}$ Florey Department of Neuroscience \\ and Mental Health; ${ }^{3}$ Department Anatomy \& Neuroscience, University of Melbourne, \\ Parkville, Victoria, 3010; ${ }^{4}$ Department of Applied Psychology, Royal Melbourne University \\ of Technology, Melbourne, Victoria, 3000, Australia
}

\title{
Communicating Authors:
}

Dr Jhodie Duncan or Professor Andrew Lawrence

Florey Institute of Neuroscience \& Mental Health,

L3, Kenneth Myer Building

Cnr Genetics Lane on Royal Parade

University of Melbourne, Parkville, Victoria, 3010, Australia

Telephone: +613 9035 6731/6692

Fax: +61309353107

Email address:

Jhodie.duncan@florey.edu.au or Andrew.Lawrence@florey.edu.au

\section{Funding:}

This study was supported by The National Health and Medical Research Council of Australia of which AJL is a Principal Research Fellow (1020737), the Australian Research Council (DP 110100379) of which JRD is a Future Fellow (100100235) and the Victorian Government's Operational Infrastructure Support Scheme. There are no conflicts of interest in this study. 


\begin{abstract}
Rationale Inhalant abuse is prevalent in adolescent populations, with chronic use resulting in neurobiological and cognitive abnormalities in adulthood. However, the nature and persistence of cognitive dysfunction, particularly following adolescent inhalant abuse, remains equivocal. Objective The present study assessed specific cognitive processes beginning in late adolescence and adulthood following adolescent inhalation of toluene, a main component of many compounds readily abused. Methods Adolescent male Wistar rats (postnatal day (PN) 27) were exposed to chronic intermittent inhaled toluene (10,000 ppm) for $1 \mathrm{hr} /$ day, 3 days/week for 4 weeks (PN 27 - 52) to mimic the patterns observed in human adolescent inhalant abusers. Following toluene exposure, motor and cognitive function was assessed. Results Adolescent toluene exposure did not alter motor learning in the Rotarod task (PN 58), or acquisition, reversal or retention of spatial learning in the Morris water maze (PN 55 - 64). In contrast, it delayed acquisition of instrumental responding for sucrose $(5 \% \mathrm{w} / \mathrm{v})$, and impaired operant reversal learning and cue-induced reinstatement of sucrose-seeking in adulthood (PN 57 - 100). Conclusion This study demonstrates that exposure to toluene at an abuse concentration during adolescence results in specific impairments in aspects of instrumental learning, without altering motor function and spatial learning in late adolescence/early adulthood. Our data imply that persistent alterations in reward processing may occur following adolescent inhalant misuse.
\end{abstract}

Keywords: Inhalant Abuse, Cognition, Operant Self-administration, Sucrose 


\section{Introduction}

Inhalation of volatile organic solvents, such as toluene, is a significant public health concern particularly within adolescent populations. Inhalants are commonly the initial "drug" experienced as they are cheap, legal and readily accessible providing a rapid intoxicating effect (Lubman et al. 2008). Notably, adolescent inhalant abuse is a predictor of substance abuse disorders later in life, and has a significant co-morbidity with neuropsychiatric disorders, the likelihood of which is associated with age of abuse onset (Wu et al. 2004). Furthermore, adolescence is an integral maturational period in which dynamic reorganisation in white and grey matter structures occurs, particularly within regions associated with cognitive and executive functions, such as the prefrontal cortex (PFC) (Spear 2000). Consequently, the negative implications of adolescent inhalant abuse are thought to relate to the vulnerability of the maturing brain during this period (Lubman et al. 2007).

Chronic inhalant abuse results in significant neuropathological abnormalities, with human imaging studies reporting both white (Yamanouchi et al. 1995, Rosenberg et al. 2002), and grey matter lesions (Aydin et al. 2009). These abnormalities are correlated with neurological and cognitive deficits, such as ataxia, tremors, learning and memory deficits, as well as impaired visuospatial, verbal and executive functions (Rosenberg et al. 2002, Aydin et al. 2009). The severity of these motor and cognitive deficits is associated with both the duration of abuse and the severity of neuropathological abnormalities (Yucel et al. 2008). While some degree of recovery may occur with abstinence in humans, deficits in learning and memory and executive function often persist (Cairney et al. 2005, Dingwall et al. 2011), even following protracted abstinence (Cairney et al. 2013). Therefore, neural circuits mediating cognitive and executive functions may be susceptible to persistent inhalant-induced impairments.

The volatile organic solvent toluene is found in many household products that are commonly abused. Toluene has high potential for abuse as it can elicit a conditioned place preference in rodents (Funada et al. 2002) and is self-administered in mice (Blokhina et al. 
2004) and non-human primates (Weiss et al. 1979). Similar to other drugs of abuse, toluene's ability to regulate signalling within the mesocorticolimbic dopaminergic (DA) system is thought to underlie its acute hedonic and reinforcing properties (Lubman et al. 2008, Beckley et al. 2013). Toluene also induces neurobiological alterations within the medial PFC (mPFC) (Williams et al. 2005), and animal studies have demonstrated that toluene exposure can result in cognitive impairments in tasks such as novel object recognition (Lin et al. 2010, Huerta-Rivas et al. 2012), inhibitory avoidance (Huerta-Rivas et al. 2012), spatial learning (von Euler et al. 1993) and instrumental learning (Bowen and McDonald 2009). Importantly, the negative implications of adolescent toluene inhalation remain relatively unknown. Few animal studies have investigated the long-term effects of chronic adolescent toluene exposure upon locomotor activity (Batis et al. 2010), cognitive function (Lin et al. 2010, Huerta-Rivas et al. 2012), or exposure to concentrations that model human abuse settings (> 5,000 ppm) (Bowen and McDonald 2009). This is further impacted by the fact that adolescents display differential sensitivity to inhalants compared to adults in animal models (O'Leary-Moore et al. 2009 Batis et al. 2010).

The present study therefore aimed to investigate the impact of adolescent chronic intermittent toluene (CIT) exposure at an abuse concentration (10,000 ppm) upon specific aspects of motor, spatial and reward-based learning in late adolescence and adulthood. Following a period of adolescent air (controls) or CIT exposure, separate cohorts of rats were tested in the rotarod, morris water maze, or operant self-administration paradigms to investigate discrete, as opposed to general, cognitive processes. We hypothesised that adolescent CIT exposure would impair specific learning and memory processes due to the maturational vulnerability of neural circuitry during this period. 


\section{Materials and Methods}

Animals

Adolescent male Wistar rats (PN 24; Air-exposed, $\mathrm{n}=39$; CIT-exposed, $\mathrm{n}=41$ ) were obtained from the Animal Resources Centre (Perth, Australia). In rats, adolescence ranges from weaning at PN 21 to adulthood at PN 60 (Spear 2000). Rats were pair housed, maintained on a $12 \mathrm{hr}$ light/dark cycle (lights on at 7:00) and given access to food and water ad libitum. Rats were acclimatised for 3 days prior to any experimental manipulation. All experiments were performed in accordance with the Prevention of Cruelty to Animals Act, 1986 under the guidelines of the National Health and Medical Research Council Code of Practice for the Care and Use of Animals for Experimental Purposes in Australia.

Toluene inhalation exposure

Exposure to toluene inhalation was conducted as previously described (Duncan et al. 2012). Briefly, rats were acclimatised to the laboratory at least $1 \mathrm{hr}$ prior to exposure to toluene or air during which time their body weights were recorded. Exposure to vapourised toluene was conducted in specialised chambers $(4.76 \mathrm{~L} ; 17.6 \times 16.5 \times 16.4 \mathrm{~cm})$ connected to a toluene vapour system whereby air was pumped through liquid toluene (1.08389, purity 99.8\%, Merck, Vic, Australia) in a gas wash-bottle to produce toluene vapour. Flow meters allowed the regulation of the desired concentration of toluene vapour in the exposure chambers and the concentration verified using a previously calibrated inline gas chromatography system (Shimadzu Corporation, Kyoto, Japan). A minimum of 3 readings were taken per session with deviations greater than $100 \mathrm{ppm}$ of the desired toluene concentration being corrected. Chambers of similar design but exposed to room air only were utilised for control animals ( 0 ppm exposure).

Three separate experimental cohorts were assessed (Rotarod, Morris water maze, and operant self-administration of sucrose) and behavioural testing was conducted such that each cohort was tested specifically in a single behavioural paradigm. CIT-exposed rats in the Rotarod cohort were also subjected to assessment of locomotor activity during acute recovery following the initial and final exposure to toluene. Within each experimental 
cohort rats were randomly assigned to inhale either air or toluene (10,000 ppm) for $1 \mathrm{hr}$ per day, 3 days per week (Monday, Wednesday, Friday), for 4 weeks. Thus, the 4 week exposure period took animals from early (PN 27) to late adolescence (PN 52). The chronic intermittent exposure paradigm employed was designed to mirror the human pattern of toluene abuse at an equivalent abuse concentration (Lubman et al. 2008) where the sensitivity to toluene is approximately equivalent in both species (Benignus et al. 2007). After $1 \mathrm{hr}$ of exposure, rats were placed back into their home cages and semi isolated from other rats for at least $1 \mathrm{hr}$ to avoid the possible confounds of olfactory stimulation by toluene scent on the fur. All chambers were briefly cleaned with $70 \%$ ethanol between sessions. Exposures were conducted at room temperature $\left(\sim 21^{\circ} \mathrm{C}\right)$ under normal lighting and each rat was exposed at approximately the same time each day $(\sim 9: 00-11: 00$ or $2-4$ hrs into the light cycle).

\section{Locomotor Recovery and Rotarod}

To assess recovery from the acute locomotor effects of toluene inhalation, CITexposed rats $(n=10)$ were placed in locomotor cells $\left(\operatorname{TruScan}^{\mathrm{TM}}\right.$ Photobeams; Coulbourn Instruments, Allentown, PA, USA) immediately following the initial and final exposure and horizontal (HP) and vertical plane (VP) activity recorded for 40 mins as previously described (Duncan et al. 2013). Motor learning and co-ordination following adolescent CIT exposure was then assessed. Both air- $(n=7)$ and CIT-exposed rats $(n=10)$ were tested on the Rotarod 6 days after the last exposure (PN 58). The Rotarod apparatus (Ugo Basile, Italy) consisted of a tactile rod $6 \mathrm{~cm}$ in diameter, separated via partitions into four segments $8.7 \mathrm{~cm}$ wide. Two training sessions 15 mins apart, one at a fixed speed of $4 \mathrm{rpm}$ and one accelerating from $4 \mathrm{rpm}$ to $24 \mathrm{rpm}$, were undertaken whereby each rat had to remain on the Rotarod for 2 mins. Following a $4 \mathrm{hr}$ retention period rats were subjected to three $5 \mathrm{~min}$ tests 15 mins apart on an accelerating rod ( $4 \mathrm{rpm}$ to $24 \mathrm{rpm}$ ) and the latency to fall was recorded. Motor co-ordination was quantified as increased time spent on the Rotarod prior to falling.

\section{Morris water maze}


Spatial learning was assessed in the Morris water maze following 4 weeks of air (n $=22)$ or CIT exposure $(\mathrm{n}=23)$. The water maze $(180 \mathrm{~cm}$ diameter x $62 \mathrm{~cm}$ depth $)$ was filled with $42 \mathrm{~cm}$ of water $\left(25^{\circ} \mathrm{C}\right)$ and divided in notional quadrants with distinct visual cues positioned at the North, South, East, and West points of the pool to allow spatial reference learning to occur (Featherby et al. 2008). Training consisted of four 2 min trials per day with 30 min intervals between each trial, which commenced 3 days following the final exposure day. Rats were placed on the edge of the maze facing the wall in one of the four quadrants (different each day) and allowed to locate the submerged platform or, failing this, they were then guided to its location. Rats were allowed to remain on the platform for 30 secs and then removed from the maze. Throughout acquisition (5 days, PN $55-59$ ), the platform was positioned within the South East quadrant (Fig. 3a). To assess reversal learning in this paradigm, the platform location was positioned in the North West quadrant on day 6 and day 7 (PN $60-61$ ). On day 8, the platform location was relocated to the North East quadrant and remained in this location on day 9 (PN $62-63)$. Following reversal learning, retention of spatial memory was assessed in a single 1 min probe test on day 10 (PN 64). In this trial, the platform was removed from the pool and the time spent within each quadrant assessed. For all experimentation, tracking data was obtained with EthoVision 3.0 (Noldus Information Technology, Sterling, VA).

\section{Operant self-administration of sucrose}

Three days after the 4 week exposure period, all rats (Air $n=10$; CIT $n=10)$ were trained to respond for sucrose $(5 \% \mathrm{w} / \mathrm{v})$ in standard sound-attenuated operant chambers (Med Associates, VT, USA), essentially as previously described (Jupp et al. 2011). Briefly, operant chambers were equipped with 2 retractable levers on either side of the chamber adjacent to a fluid receptacle. Availability of sucrose was conditioned with the presence of a discriminative olfactory cue (vanilla essence placed directly underneath the active lever), and fulfilment of a fixed ratio (FR) requirement resulted in sucrose delivery paired with 1 sec illumination of a cue light (conditioned stimulus (CS)) located above the active lever. 
Both active and inactive lever responses within each session were recorded with each delivery consisting of $100 \mu \mathrm{l}$ of either sucrose or water respectively. Rats were trained to respond for sucrose in a single overnight session $(14 \mathrm{hrs})$ on an FR2 schedule of reinforcement followed by daily 20 min operant sessions on an FR3 schedule of reinforcement, 5 days per week.

Acquisition consisted of 10 operant sessions (PN 57 - 70) such that lever discrimination and stable responding was established $(>30$ active lever presses and $<15 \%$ variability over last 3 sessions) (Fig. 3a). Failure to reach these criteria resulted in exclusion from data analysis. Two CIT-exposed rats were excluded, one due to illness throughout the self-administration paradigm, while the other failed to reach the acquisition criteria (CIT $n=$ 8). Following acquisition, rats were tested under a progressive ratio (PR) schedule of reinforcement in two 90 min sessions as previously described (Jupp et al. 2011), where the response requirement increased by 1 upon each reward delivery. PR sessions were interspersed with a single FR3 session (ie over a 3 day period; PN 71 - 73). Breakpoint was defined as the maximum rewards received, which was equal to the maximum PR requirement fulfilled. Following the second PR session, reacquisition of lever responding occurred over 2 days in 20 min FR3 sessions followed by instrumental extinction in daily 20 min sessions whereby the discriminative cue was removed from the chambers, and responding on either lever had no programmed consequences. Extinction continued for 10 consecutive days (PN $78-87)$ to allow rats to reach extinction criteria $(<10$ active lever presses or $<15 \%$ of the last FR3 session over the final 3 days of extinction). The day following extinction, cue-induced reinstatement of sucrose-seeking was assessed in a single 20 min session (PN 88) whereby lever responding was precipitated by the reintroduction of the discriminative cue under the active lever, and contingent CS presentation upon each FR3 response. A single sucrose delivery $(<100 \mu \mathrm{l})$ was received upon the first FR3 response on the active lever, with no further deliveries upon subsequent responding. A single air-exposed rat was excluded due to technical failure of the operant chamber $(n=9)$. After the single reinstatement test, reacquisition of active lever responding for sucrose 
occurred over 2 days in 20 min FR3 sessions. Following reacquisition, reversal of lever locations was employed to assess reversal learning (PN 92 - 100). Reversal sessions did not differ from former FR3 sessions, except the lever locations were reversed and the discriminative cue was positioned under the newly located active lever. Once responding was reacquired to pre-reversal levels (5 days), the levers were again reversed back to their original locations to assess performance upon a second reversal of lever locations.

\section{Statistical Analyses}

Statistical analyses were performed using IBM SPSS Statistics 20 (IBM, USA), and SigmaStat 3.5 (Jandel, San Jose, CA, USA) and all graphs were produced with GraphPad Prism 6 (GraphPad Software, San Diego, CA, USA). Body weights throughout exposure were analysed using two-way repeated measures Analysis of Variance (RM ANOVA) with treatment and time as factors. Time course of locomotor recovery following exposure in CIT-exposed rats was analysed with a two-way RM ANOVA with exposure day and time as factors; paired comparisons were employed. Rotarod data was analysed using a two-way RM ANOVA with treatment and trial as factors. Water maze data were analysed with twoway RM ANOVAs with treatment and day as factors for acquisition, and reversal sessions (each reversal session analysed individually in comparison to pre-reversal day) and treatment and quadrant as factors for retention trial data; paired comparisons were employed. For all operant self-administration studies, two-way RM ANOVAs were used to analyse acquisition (treatment $\mathrm{x}$ session), lever discrimination (treatment $\mathrm{x}$ lever), total lever responses (treatment $\mathrm{x}$ lever) and time courses (treatment $\mathrm{x}$ time) for reversal data and total lever responses (treatment $\mathrm{x}$ session) and time courses (treatment $\mathrm{x}$ time) of cue-induced reinstatement data. Holm-Sidak paired comparisons were utilised for all post hoc comparisons. Unpaired $t$ tests were employed to analyse the difference between groups in body weights upon cessation of experimentation, total swim distance in the retention trial of water maze experiments, as well as lever responses and breakpoints in PR sessions. All data are expressed as mean \pm SEM, or mean of means \pm SEM (water maze acquisition and reversal data). Significance was accepted as $p<0.05$. 


\section{Results}

Effect of adolescent CIT exposure on body weight and recovery of motor function

Throughout the 4 week exposure period toluene significantly attenuated weight gain of CIT- compared to air-exposed rats in all cohorts assessed. Analysis of grouped body weights throughout the exposure period revealed a main effect of treatment $\left(\mathrm{F}_{(1,77)}=31.94\right.$, $p<0.001)$ and time $\left(\mathrm{F}_{(2,154)}=10364.40, p<0.001\right)$, plus a significant treatment $\mathrm{x}$ time interaction $\left(\mathrm{F}_{(2,154)}=125.62, p<0.001\right.$, Fig. 1a). Post hoc analysis revealed that body weights were reduced in CIT-exposed rats from the $6^{\text {th }}$ exposure day $(p<0.001)$. Body weights remained significantly reduced in CIT-exposed rats at the cessation of experimentation in both the Rotarod (Air; $352.1 \pm 8.6$ g; CIT $313.9 \pm 9.4 \mathrm{~g}, p<0.01$; PN 58) and Morris water maze cohorts (Air; $384.8 \pm 4.1 \mathrm{~g}$; CIT $333.9 \pm 4.1 \mathrm{~g}, p<0.001 \mathrm{PN}$ 64). While there was a trend towards decreased body weight in CIT- compared to airexposed rats upon cessation of experimentation in the sucrose operant cohort, this did not reach statistical significance (Air 568.6 $\pm 21.3 \mathrm{~g}$; CIT $518.6 \pm 13.9 \mathrm{~g}, p=0.08$; PN 100).

Analysis of HP activity during recovery following the initial (PN 27) and final toluene exposure (PN 52) revealed a main effect of exposure $\left(\mathrm{F}_{(1,18)}=32.59, p<0.001\right)$ and time $\left(\mathrm{F}_{(9,162)}=10.90, p<0.001\right)$ as well as an exposure $\mathrm{x}$ time interaction $\left(\mathrm{F}_{(9,162)}=3.695, p\right.$ $<0.001$, Fig. 1b). Post hoc analysis revealed significantly increased HP activity from $16-$ 32 mins, and at 40 mins recovery $(p<0.01)$ on the final compared to the initial exposure suggesting enhanced recovery from the hypolocomotor effects of toluene by the end of the exposure period. No differences in VP activity were observed between the initial and final exposure (data not shown).

Adolescent CIT exposure does not impair motor learning or co-ordination

Analysis of Rotarod performance 6 days following the final exposure day (PN 58) revealed a main effect of trial $\left(\mathrm{F}_{(2,30)}=15.39, p<0.001\right)$ but no main effect of treatment $(\mathrm{F}$ $<1)$ or a factors interaction $(\mathrm{F}=1.12$, Fig. 2$)$. Subsequent post hoc analysis revealed that both air- and CIT-exposed rats displayed significantly increased time on the Rotarod across 
the 3 trials $(p<0.05$, Fig. 2). These results demonstrate that adolescent CIT exposure did not affect motor learning or co-ordination as measured by Rotarod performance.

Spatial learning and spatial reversal learning remains intact following adolescent CIT exposure

Analysis of the latency to discover the submerged platform within the acquisition phase of the Morris water maze experiment (PN 55 - 59; Fig. 3a) revealed a significant main effect of day $\left(\mathrm{F}_{(4,172)}=80.30, p<0.001\right)$, with no main effect of treatment or a factors interaction $(\mathrm{F}<1)$, suggesting that both groups similarly acquired the task (Fig. 3b). Following acquisition, spatial reversal learning was tested over the next 4 days (PN $60-63$ ) whereby the platform location was relocated twice ( 2 days per location). Analysis of latencies to find the submerged platform on the pre-reversal (day 5) and initial reversal day (R1, day 6) revealed a main effect of day $\left(\mathrm{F}_{(1,43)}=14.66, p<0.001\right)$ with no main effect of treatment or a factors interaction ( $\mathrm{F}<1$, Fig. 3c left panel). The second reversal (R2) revealed no main effects or a factors interaction $(\mathrm{F}<1$, Fig. $3 \mathrm{c}$ right panel). Retention of spatial memory was then assessed in a single 1 min probe test (day 10, PN 64), whereby the submerged platform was removed and the time spent within each quadrant recorded. Analysis of the probe test data revealed a main effect of quadrant $\left(\mathrm{F}_{(3,129)}=37.32, p<\right.$ 0.001 ) with no main effect of treatment or a factors interaction (F $<1$, Fig. 3d). Post hoc analysis revealed significantly greater time spent in the North East quadrant where the platform was located on the previous day $(p<0.001)$ and significantly less time spent in the South West quadrant where the platform was never located $(p<0.001)$, with no differences between treatment groups (Fig. 3d). Total distance travelled during the probe test did not differ between groups (Fig. 3e). These results suggest that the acquisition, reversal and retention of a spatial learning task were not affected following adolescent CIT exposure under the current paradigm conditions.

Acquisition but not expression of instrumental responding for sucrose is impaired following adolescent CIT exposure 
For sucrose operant self-administration experiments (Fig. 4a) analysis of the initial acquisition of active lever responding for sucrose (operant sessions $1-3$, PN 57 - 59; Fig. $4 \mathrm{~b})$, revealed a main effect of session $\left(\mathrm{F}_{(2,32)}=7.15, p<0.01\right)$, with no main effect of treatment $(\mathrm{F}=2.04)$ or a factors interaction $(\mathrm{F}=2.44)$. Post hoc analysis revealed a significant increase of active lever responding across the first 3 operant sessions in air- $(p<$ 0.001 ) but not CIT-exposed rats ( $p=0.39$, Fig. 4b). However, analysis of lever discrimination on the final acquisition day (operant session 10, PN 70) revealed a main effect of lever $\left(\mathrm{F}_{(1,16)}=37.16, \mathrm{p}<0.001\right)$, with no main effect of treatment or a factors interaction $(\mathrm{F}<1)$, suggesting that once acquired, there was no significant difference in stable responding for sucrose between groups (Fig. 4c).

Motivation to acquire sucrose was not affected following adolescent CIT exposure

Following acquisition, we employed a PR schedule of reinforcement to test motivation to acquire a sucrose reward in CIT-exposed rats. Analysis of mean active lever responding (Fig. 4d) and breakpoints (Fig. 4e) revealed no significant differences between groups (air or CIT, PN $71-73$ ). These results suggest that the motivation to obtain and consume sucrose was not affected following adolescent CIT exposure.

\section{Extinction and cue-induced reinstatement}

Analysis of active lever responding throughout extinction training (PN $78-87$ ) revealed a gradual decrease in active lever responding across days as evidenced by a main effect of day $\left(\mathrm{F}_{(9,144)}=65.23, p<0.001\right)$, with no main effect of treatment or a factors interaction $(\mathrm{F}<1$; data not shown). Once extinction criteria were met, all rats were subjected to cue-induced reinstatement to assess the effect of adolescent CIT exposure upon reward-seeking behaviour (PN 88). Analysis of total active lever responses upon cueinduced reinstatement of sucrose-seeking (Fig. 5a), revealed a main effect of session $\left(\mathrm{F}_{(1,15)}\right.$ $=39.87, p<0.001$ ), as both treatment groups displayed a significant increase of active lever responding upon presentation of cues previously associated with reward delivery, yet there was no main effect of treatment $(F<1)$ or a factors interaction $(F=1.69)$. However, analysis of the time course of active lever responding within the reinstatement session (Fig. 
$5 b)$, revealed a main effect of time $\left(\mathrm{F}_{(3,45)}=21.03, p<0.001\right)$ and a treatment $\mathrm{x}$ time interaction $\left(\mathrm{F}_{(3,45)}=3.03, p<0.05\right)$. Subsequent post hoc analysis revealed significantly lower active lever responding within the first 5 mins of the reinstatement session in CITcompared to air-exposed rats ( $p<0.01$, Fig. 5b). Furthermore, CIT-exposed rats displayed a significant increase in the latency to fulfil an initial FR3 response ( $p<0.05$, Fig. 5c).

Operant reversal learning is impaired following adolescent CIT exposure

Operant reversal learning was assessed following reacquisition of active lever responding after cue-induced reinstatement ( 2 days). Analysis of total lever responses in the initial reversal session (R1, PN 92; Fig. 6a, left panel) revealed no main effects or a factors interaction $(\mathrm{F}<1)$. However, analysis of the time course of active lever responses within R1 (Fig. 6b) revealed a main effect of time $\left(\mathrm{F}_{(3,48)}=7.486, p<0.001\right)$ and a treatment $\mathrm{x}$ time interaction $\left(\mathrm{F}_{(3,48)}=3.71, p<0.05\right)$. Subsequent post hoc analysis revealed that air-exposed rats significantly increased active lever responding throughout the session $(p<0.05)$, while CIT-exposed rats displayed no significant differences in active lever responding throughout this session (Fig. 6b). Analysis of total lever responses on the second reversal (R2, PN 98; Fig. 6a, right panel) revealed a main effect of lever $\left(\mathrm{F}_{(1,16)}=44.54, p<0.001\right)$ but no main effect of treatment or a factors interaction $(\mathrm{F}<1)$. No differences were observed in the time course of active lever responding between groups within R2 (data not shown). 


\section{Discussion}

In this study, we investigated the impact of chronic adolescent toluene inhalation at a concentration relevant to human abuse settings (10,000 ppm) (Bowen et al. 2006) upon subsequent motor and cognitive function in late adolescence and adulthood. Adolescent CIT exposure resulted in an attenuation of weight gain within the exposure period, which appeared to recover by around 7 weeks after exposure ceased (PN 100). Enhanced recovery from the acute hypolocomotor effects of toluene inhalation were also observed as CITexposed rats displayed a more rapid onset of increased HP activity during recovery following the final compared to the initial exposure as previously demonstrated in our model following a shorter duration of exposure (Duncan et al. 2013). Consistent with this, we have a previously demonstrated tolerance to the acute ataxic effects of adolescent CIT exposure in this model (Duncan et al. 2013). Rotarod performance in late adolescence revealed that both motor co-ordination and motor learning were not affected following adolescent CIT exposure. Furthermore, the acquisition, reversal and retention of spatial learning in the Morris water maze did not differ between air- and CIT-exposed rats. Together, these results suggest that both motor function and spatial learning are largely intact following adolescent CIT exposure in this model. However, operant responding for sucrose in adulthood revealed impairments in specific aspects of instrumental learning following adolescent CIT exposure. Thus, although the integrity of neural circuits was not directly assessed in this study, our results suggest that some circuits appear more sensitive than others to modulation following adolescent CIT exposure.

\section{Motor co-ordination and spatial learning are intact following adolescent CIT exposure}

Here we show that adolescent CIT exposure does not subsequently impair motor co-ordination or motor learning as evidenced by Rotarod performance in late adolescence. This is in line with studies assessing the effects of chronic exposure to low concentrations of toluene (< $1500 \mathrm{ppm}$ ) (Ladefoged et al. 1991, Beasley et al. 2012). Interestingly, adolescent CIT exposure did not impair acquisition, reversal or retention of spatial learning in the Morris water maze task, indicating that spatial learning and related memory processes 
are not impaired under our conditions. Thus, these results suggest a lack of persistent dysfunction within neural circuits involved in spatial learning, such as the hippocampus, following adolescent CIT exposure (Morris et al. 1986). In contrast, chronic low concentrations of toluene inhalation ( $80 \mathrm{ppm}, 6 \mathrm{hrs} /$ day, 5 days/week for 4 weeks) impaired Morris water maze performance (von Euler et al. 1993), which may reflect hippocampal neurotoxicity (Seo et al. 2010). However, our study is line with Ladefoged and colleagues (1991), who demonstrated that daily toluene inhalation (500 and $1500 \mathrm{ppm}$ ), for up to 6 months did not result in impaired acquisition or retention of spatial memory in the Morris water maze task. Acute toluene inhalation $(5,000 \mathrm{ppm}$ for $30 \mathrm{mins})$ alters hippocampal output in vivo, yet only impairs water maze performance when exposure occurs prior to each training session, suggesting transient effects upon hippocampal-dependent learning (Gmaz et al. 2012a, Gmaz et al. 2012b).

Discrepancies between previous studies and the present findings likely arise from the various exposure paradigms employed; we have previously demonstrated that the duration of the exposure period (4 compared to 8 weeks) and consequently the number of exposures (12 compared to 24 exposures) alters both the behavioural and neuropathological consequences of toluene inhalation (Duncan et al. 2012). Indeed, duration of abuse history appears to relate to the neuropathological (Yamanouchi et al. 1995, Aydin et al. 2002) and cognitive abnormalities (Cairney et al. 2005) observed in human inhalant abusers. Thus, the exposure paradigm we employed does not appear to result in generalised deficits in learning and memory processes, arguing against overt neurotoxicity or dysfunction in regions required for motor learning and co-ordination or spatial learning.

Adolescent CIT exposure results in specific impairments in aspects of instrumental learning

The implications of chronic adolescent toluene exposure on subsequent instrumental learning remains relatively unknown, although studies have assessed the effects of both acute and chronic toluene inhalation on reinforced operant responding in adult animals (Bowen et al. 2006). Adolescent CIT exposure delayed the initial acquisition of instrumental responding for sucrose. However, after acquisition no differences were 
observed between air- or CIT-exposed rats suggesting that expression of instrumental responding was intact. In line with these findings, daily toluene inhalation (1000 ppm) for 13 weeks, resulted in delayed acquisition of instrumental responding in a simple signal detection task, where no deficits were observed once the task was acquired (Beasley et al. 2012). Furthermore, Bowen and McDonald, 2009, demonstrated that daily toluene inhalation (3,600 and 6,000 ppm for 40 days) following initial operant training, impaired responding for sweetened condensed milk in an instrumental "wait for reward" task, where performance was impaired throughout the exposure period (testing $22-23$ hrs following daily exposures), as well as up to 40 days of recovery in mice exposed to $6,000 \mathrm{ppm}$. The latter study suggests that following training, inhalation of toluene may persistently impair retention/retrieval of a previously learnt instrumental task, although potential motor deficits or amotivation may also account for these impairments (Bowen and McDonald 2009). In our study, motor deficits were not observed and responding on PR schedules did not differ between air- and CIT-exposed rats suggesting that the motivation to acquire sucrose was similar between groups and therefore should not confound initial responding in CITexposed rats.

Although reversal learning deficits were not observed in the Morris water maze, reversal of lever locations in operant experiments revealed that adolescent CIT exposure modestly impaired operant reversal learning within the initial session (R1). This was evident as air- but not CIT-exposed rats significantly increased responding on the newly located active lever throughout this session. This impairment was observed 40 days following the final toluene exposure yet was not evident in subsequent reacquisition sessions or a second reversal session (R2). Badanich et al., 2011, demonstrated that posttraining chronic intermittent ethanol vapour inhalation, resulted in a transient deficit in reversal learning present at $72 \mathrm{hrs}$ but not 14 days withdrawal, with no effects on an $\mathrm{mPFC}-$ mediated set-shifting task, suggestive of preferential vulnerability of the orbitofrontal cortex (OFC). Reversal-specific impairments have also been observed following cocaine (Calu et al. 2007, Schoenbaum et al. 2004) and methamphetamine administration (Izquierdo et al. 
2010). However, lesions of the OFC result in perseverative errors (ie failure to inhibit responding to previously rewarded stimulus) in reversal learning tasks (Schoenbaum et al. 2002, Chudasama and Robbins 2003), which were not observed in the current study. Thus, it is unlikely that adolescent CIT exposure results in overt damage of regions implicated in reversal learning but persistent functional alterations within these regions may contribute to the initial reversal learning deficit in the present study.

While instrumental extinction was not different between groups, upon cue-induced reinstatement of sucrose-seeking, CIT exposed rats showed attenuated active lever responding early in the session. This resulted in an increased latency to achieve the initial FR3 response, and thus the initial CS presentation. As cue-induced reinstatement was precipitated by the re-introduction of the discriminative cue and contingent CS presentation, these results suggest that the excitatory effect of the discriminative cue may have been greater in air- compared to CIT-exposed rats. This may account for the potentiated responding of air- compared to CIT-exposed rats within the first 5 mins of this session. As the discriminative cue may be considered a contextual association to sucrose availability, adolescent CIT exposure may affect the acquisition of context-reward associations and/or the processing of contextual information, which may contribute to the delay in initial responding in CIT-exposed rats. Nevertheless, despite a delay in responding, CIT-exposed rats reinstated to a similar magnitude as air-exposed rats overall, arguing against overall generalised reduction in the incentive salience of the conditioned stimuli.

Adolescent CIT exposure did not alter motor or spatial learning and related memory processes, yet the specific impairments in acquisition, reversal and cue-induced reinstatement of operant responding for sucrose suggest that toluene may persistently affect the function of circuits involved in instrumental learning. Indeed, the maturational trajectory of discrete neural circuits during adolescence may attribute certain regions with a vulnerability to the effects of adolescent CIT exposure. For example, prefrontal regions continue to mature beyond the onset of early adulthood (Spear 2000, Giedd 2004), and the refinement of the mesocorticolimbic DA system, integral for instrumental learning and 
goal-directed behaviour, occurs throughout adolescence, and is particularly vulnerable to alterations during this period (Manitt et al. 2011). Consequently, toluene exposure during adolescence may affect these maturational processes resulting in persistent behavioural dysfunction in adulthood. For example a recent study demonstrated that brief toluene inhalation $(5,700 \mathrm{ppm}$ in two 10 mins exposures) in adolescent rats (PN $40-47)$ induces persistent (up to 21 days) synaptic plasticity of mesoaccumbal but not mesocortical projecting DA neurons within the VTA (Beckley et al. 2013) and that inhibition of the mPFC prior to inhalation regulated this toluene-induced plasticity. This suggests that toluene inhalation attenuates glutamatergic output from the mPFC, thereby enabling synaptic adaptation within the VTA (Beckley et al. 2013). We have also shown long-term changes to the glutamatergic system following adolescent CIT exposure presenting as altered behavioural responses to acute administration of the NMDA receptor antagonist, MK-801 (Duncan et al. 2013). While we did not directly investigate the integrity of neural circuits in this study, the behavioural deficits observed in specific aspects of operant responding for sucrose presumably reflect altered functional integrity of neural circuits mediating these behaviours.

Acquisition of instrumental responding requires functional integrity of the mPFC, which is involved in the formation, but not the expression of action-outcome (A-O) associations (Corbit and Balleine 2003, Ostlund and Balleine 2005). Thus, adolescent CIT exposure may alter the function of the $\mathrm{mPFC}$ resulting in the delayed acquisition of $\mathrm{A}-\mathrm{O}$ associations and consequently attenuate initial instrumental responding for sucrose compared to air-exposed rats. The mPFC is also required for cue elicited reward-seeking behaviours (McFarland et al. 2003, Ishikawa et al. 2008), and encoding of contextual information in operant conditioning (specifically anterior cingulate cortex) (Torregrossa et al. 2013). Thus, altered function of the mPFC in CIT-exposed rats may also contribute to the deficits observed upon cue-induced reinstatement. However, regions such as the basolateral amygdala (BLA) are also involved in cue- and context-induced reinstatement of reward-seeking as well as the acquisition of Pavlovian associations and processing of the 
incentive salience of rewards and conditioned stimuli to mediate goal-directed behaviour (Blundell et al. 2003, Lasseter et al. 2011, Balleine et al. 2003, Corbit and Balleine 2005). Thus, the behavioural deficits observed implicate several structures that may be vulnerable to adolescent CIT exposure.

\section{Limitations}

Adolescent CIT exposure attenuated weight gain within the exposure period, recovering to similar levels as air-exposed rats by 7 weeks following the exposure period (PN 100). Thus, metabolic dysfunction may have altered the incentive salience of sucrose rewards in operant self-administration experiments initially (Jarosz et al. 2008). Nevertheless, the recovery of body weight in CIT-exposed rats suggests the lack of persistent metabolic dysfunction under the present conditions. Furthermore, rats were fed ad libitum unlike many reward-based learning studies where food restriction is employed to increase the motivation to acquire food rewards (Corbit \& Balleine 2005). It must also be noted that toluene inhalation (1,000 ppm, 5 hrs/day, 5 days/week for 4 weeks), induces transient deficits in olfactory functioning associated with degenerative effects on olfactory epithelium, which recovers within $1-2$ weeks following the exposure period (Jacquot et al. 2006). Thus, adolescent CIT exposure could in theory affect olfaction, impairing the ability of CIT-exposed rats to utilise the discriminative olfactory cue to guide instrumental performance in this study. However, considering the extended duration of operant selfadministration experiments, and the lack of overall effects on responding between air- and CIT-exposed rats it appears these potential confounds are not likely to be present in the current study.

Conclusion

Adolescent CIT exposure in rats results in specific impairments in aspects of instrumental learning, without altering motor function and spatial learning in late adolescence and adulthood. These impairments were persistent, suggesting susceptibility of specific neural circuits and support the hypothesis that adolescent CIT exposure alters the 
functioning of discrete brain regions (rather than generalised "toxicity"), ultimately resulting in deficits of reward-based learning in adulthood.

1

2

3

4

5

6

7

8

9

10

11

12

13

14

15

16

17

18

19

20

21

22

23

24

25

26

27

28

29

30

31

32

33

34

35

36

37

38

39

40

41

42

43

44

45

46

47

48

49

50

51

52

53

54

55

56

57

58

59

60 


\begin{abstract}
Acknowledgements
The authors would like to thank Ms. Zakia Haque for assistance with running animals through the inhalant exposure paradigm. This study was supported by The National Health and Medical Research Council of Australia of which AJL is a Principal Research Fellow (1020737), the Australian Research Council (DP 110100379) of which JRD is a Future Fellow (100100235) and the Victorian Government's Operational Infrastructure Support Scheme.
\end{abstract}




\section{References}

Aydin K, Kircan S, Sarwar S, Okur O, Balaban E (2009) Smaller gray matter volumes in frontal and parietal cortices of solvent abusers correlate with cognitive deficits. AJNR Am J Neuroradiol 30:1922-1928.

Aydin K, Sencer S, Demir T, Ogel K, Tunaci A, Minareci O (2002) Cranial MR Findings in Chronic Toluene Abuse by Inhalation. AJNR Am J Neuroradiol 23:1173-1179.

Badanich KA, Becker HC, Woodward JJ (2011) Effects of chronic intermittent ethanol exposure on orbitofrontal and medial prefrontal cortex-dependent behaviors in mice. Behav Neurosci 125:879-891.

Balleine BW (2005) Neural bases of food-seeking: affect, arousal and reward in corticostriatolimbic circuits. Physiol Behav 86:717-730.

Balleine BW, Killcross AS, Dickinson A (2003) The effect of lesions of the basolateral amygdala on instrumental conditioning. J Neurosci 23:666-675.

Batis JC, Hannigan JH, Bowen SE (2010) Differential effects of inhaled toluene on locomotor activity in adolescent and adult rats. Pharmacol Biochem Behav 96:438448.

Beasley TE, Evansky PA, Bushnell PJ (2012) Behavioral effects of sub-acute inhalation of toluene in adult rats. Neurotoxicol Teratol 34:83-89.

Beckley JT, Evins CE, Fedarovich H, Gilstrap MJ, Woodward JJ (2013) Medial prefrontal cortex inversely regulates toluene-induced changes in markers of synaptic plasticity of mesolimbic dopamine neurons. J Neurosci 33:804-813.

Benignus VA, Boyes WK, Kenyon EM, Bushnell PJ (2007) Quantitative comparisons of the acute neurotoxicity of toluene in rats and humans. Toxicol Sci 100:146-155.

Blokhina EA, Dravolina OA, Bespalov AY, Balster RL, Zvartau EE (2004) Intravenous self-administration of abused solvents and anesthetics in mice. Eur J Pharmacol 485:211-218.

Blundell P, Hall G, Killcross S (2003) Preserved sensitivity to outcome value after lesions of the basolateral amygdala. J Neurosci 23:7702-7709. 
Bowen SE, Batis JC, Peaz-Martinez N, Cruz SL (2006) The last decade of solvent research in animal models of abuse: Mechanistic and behavioural studies. Neurotoxicol Teratol 28:636-647.

Bowen SE, McDonald P (2009) Abuse pattern of toluene exposure alters mouse behavior in a waiting-for-reward operant task. Neurotoxicol Teratol 31:18-25.

Cairney S, Maruff P, Burns CB, Currie J, Currie BJ (2005) Neurological and cognitive recovery following abstinence from petrol sniffing. Neuropsychopharmacology 30:1019-1027.

Cairney S, N OC, Dingwall KM, Maruff P, Shafiq-Antonacci R, Currie J, Currie BJ (2013) A prospective study of neurocognitive changes 15 years after chronic inhalant abuse. Addiction 108:1107-1114.

Calu DJ, Stalnaker TA, Franz TM, Singh T, Shaham Y, Schoenbaum G (2007) Withdrawal from cocaine self-administration produces long-lasting deficits in orbitofrontaldependent reversal learning in rats. Learn Mem 14:325-328.

Chudasama Y, Robbins TW (2003) Dissociable contributions of the orbitofrontal and infralimbic cortex to pavlovian autoshaping and discrimination reversal learning: further evidence for the functional heterogeneity of the rodent frontal cortex. J Neurosci 23:8771-8780.

Corbit LH, Balleine BW (2003) The role of prelimbic cortex in instrumental conditioning. Behav Brain Res 146:145-157.

Corbit LH, Balleine BW (2005) Double dissociation of basolateral and central amygdala lesions on the general and outcome-specific forms of pavlovian-instrumental transfer. J Neurosci 25:962-970.

Dingwall KM, Maruff P, Fredrickson A, Cairney S (2011) Cognitive recovery during and after treatment for volatile solvent abuse. Drug Alcohol Depend 118:180-185

Duncan JR, Dick AL, Egan G, Kolbe S, Gavrilescu M, Wright D, Lubman DI, Lawrence AJ (2012) Adolescent toluene inhalation in rats affects white matter maturation with the potential for recovery following abstinence. PloS one 7:e44790. 
Duncan JR, Gibbs SJ, Lawrence AJ (2013) Chronic intermittent toluene inhalation in adolescent rats alters behavioural responses to amphetamine and MK801. Eur Neuropsychopharmacol. In press, doi: 10.1016/j.euroneuro.2013.06.001

Featherby T, van den Buuse M, Lubman DI, Lawrence AJ (2008) Persistent downregulation of hippocampal CREB mRNA parallels a Y-maze deficit in adolescent rats following semi-chronic amphetamine administration. Br J Pharmacol 154:417-428.

Funada M, Sato M, Makino Y, Wada K (2002) Evaluation of rewarding effect of toluene by the conditioned places preference procedure in mice. Brain Res Protoc 10:47-54.

Gerasimov MR, Schiffer WK, Marstellar D, Ferrieri R, Alexoff D, Dewey SL (2002) Toluene inhalation produces regionally specfifc changes in the extracellular dopamine. Drug Alcohol Depend 65:243-251.

Giedd JN (2004) Structural magnetic resonance imaging of the adolescent brain. Ann N Y Acad Sci 1021:77-85.

Gmaz JM, Matthews BA, McKay BE (2012a) Toluene inhalation modulates dentate gyrus granule cell output in vivo. Neurotoxicol Teratol 34:403-412.

Gmaz JM, Yang L, Ahrari A, McKay BE (2012b) Binge inhalation of toluene vapor produces dissociable motor and cognitive dysfunction in water maze tasks. Behav Pharmacol 23:669-677.

Huerta-Rivas A, Lopez-Rubalcava C, Sanchez-Serrano SL, Valdez-Tapia M, Lamas M, Cruz SL (2012) Toluene impairs learning and memory, has antinociceptive effects, and modifies histone acetylation in the dentate gyrus of adolescent and adult rats. Pharmacol Biochem Behav 102:48-57.

Ishikawa A, Ambroggi F, Nicola SM, Fields HL (2008) Contributions of the amygdala and medial prefrontal cortex to incentive cue responding. Neuroscience 155:573-584.

Izquierdo A, Belcher AM, Scott L, Cazares VA, Chen J, O'Dell SJ, Malvaez M, Wu T, Marshall JF (2010) Reversal-specific learning impairments after a binge regimen of methamphetamine in rats: possible involvement of striatal dopamine. Neuropsychopharmacology 35:505-514. 
Jacquot L, Pourie G, Buron G, Monnin J, Brand G (2006) Effects of toluene inhalation exposure on olfactory functioning: behavioral and histological assessment. Toxicol Lett 165:57-65.

Jarosz PA, Fata E, Bowen SE, Jen KL, Coscina DV (2008) Effects of abuse pattern of gestational toluene exposure on metabolism, feeding and body composition. Physiol Behav 93:984-993.

Jupp B, Krivdic B, Krstew E, Lawrence AJ (2011) The orexin(1) receptor antagonist SB334867 dissociates the motivational properties of alcohol and sucrose in rats. Brain Res 1391:54-59.

Ladefoged O, Strange P, Moller A, Lam HR, Ostergaard G, Larsen JJ, Arlien-Soborg P (1991) Irreversible effects in rats of toluene (inhalation) exposure for six months. Pharmacol Toxicol 68:384-390.

Lasseter HC, Wells AM, Xie X, Fuchs RA (2011) Interaction of the basolateral amygdala and orbitofrontal cortex is critical for drug context-induced reinstatement of cocaine-seeking behavior in rats. Neuropsychopharmacology 36:711-720.

Lin BF, Ou MC, Chung SS, Pang CY, Chen HH (2010) Adolescent toluene exposure produces enduring social and cognitive deficits in mice: an animal model of solvent-induced psychosis. World J Biol Psychiatry 11:792-802.

Lubman DI, Yucel M, Hall WD (2007) Substance use and the adolescent brain: A toxic combination. J Psychopharmacol 21:792-794.

Lubman DI, Yucel M, Lawrence AJ (2008) Inhalant abuse among adolescents: neurobiological considerations. Br J Pharmacol 154:316-326.

Manitt C, Mimee A, Eng C, Pokinko M, Stroh T, Cooper HM, Kolb B, Flores C (2011) The Netrin Receptor DCC Is Required in the Pubertal Organization of Mesocortical Dopamine Circuitry. J Neurosci 31:8381-8394.

McFarland K, Lapish CC, Kalivas PW (2003) Prefrontal glutamate release into the core of the nucleus accumbens mediates cocaine-induced reinstatement of drug-seeking behavior. J Neurosci 23:3531-3537. 
Morris RG, Hagan JJ, Rawlins JN (1986) Allocentric spatial learning by hippocampectomised rats: a further test of the "spatial mapping" and "working memory" theories of hippocampal function. Q J Exp Psychol B 38:365-395.

O'Leary-Moore SK, Galloway MP, McMechan AP, Irtenkauf S, Hannigan JH, Bowen SE (2009) Neurochemical changes after acute binge toluene inhalation in adolescent and adult rats: A high-resolution magnetic resonance spectroscopy study. Neurotoxicol Teratol 31:382-389.

Ostlund SB, Balleine BW (2005) Lesions of medial prefrontal cortex disrupt the acquisition but not the expression of goal-directed learning. J Neurosci 25:7763-7770.

Robbins TW, Everitt BJ (1996) Neurobehavioural mechanisms of reward and motivation. Curr Opin Neurobiol 6:228-236.

Rosenberg NL, Grigsby J, Dreisbach J, Busenbark D, Grigsby P (2002) Neuropsychologic impairment and MRI abnormalities associated with chronic solvent abuse. J Toxicol Clin Toxicol 40:21-34.

Schoenbaum G, Nugent SL, Saddoris MP, Setlow B (2002) Orbitofrontal lesions in rats impair reversal but not acquisition of go, no-go odor discriminations. Neuroreport 13:885-890.

Schoenbaum G, Saddoris MP, Ramus SJ, Shaham Y, Setlow B (2004) Cocaine-experienced rats exhibit learning deficits in a task sensitive to orbitofrontal cortex lesions. Eur J Neurosci 19:1997-2002.

Seo HS, Yang M, Song MS, Kim JS, Kim SH, Kim JC, Kim H, Shin T, Wang H, Moon C (2010) Toluene inhibits hippocampal neurogenesis in adult mice. Pharmacol Biochem Behav 94:588-594.

Spear LP (2000) The adolescent brain and age-related behavioral manifestations. Neurosci Biobehav Rev 24:417-463.

Torregrossa MM, Gordon J, Taylor JR (2013) Double dissociation between the anterior cingulate cortex and nucleus accumbens core in encoding the context versus the content of pavlovian cocaine cue extinction. J Neurosci 33:8370-8377. 
von Euler G, Ogren SO, Li XM, Fuxe K, Gustafsson JA (1993) Persistent effects of subchronic toluene exposure on spatial learning and memory, dopamine-mediated locomotor activity and dopamine D2 agonist binding in the rat. Toxicology 77:223232.

Weiss B, Wood RW, Macys DA (1979) Behavioral toxicology of carbon disulfide and toluene. Environ Health Perspect 30:39-45.

Williams JM, Stafford D, Steketee JD (2005) Effects of repeated inhalation of toluene on ionotropic GABA A and glutamate receptor subunit levels in rat brain. Neurochem Int 46:1-10.

Wu LT, Pilowsky DJ, Schlenger WE (2004) Inhalant abuse and dependence among adolescents in the United States. J Am Acad Child Adolesc Psychiatry 43:12061214.

Yamanouchi N, Okada S-i, Kodama K, Hirai S, Hiroshi S, Murakami A, Komatsu N, Sakamot T, Sato T (1995) White Matter Changes Caused by Chronic Solvent Abuse. AJNR AM J Neuroradiol 16:1643-1649.

Yucel M, Takagi M, Walterfang M, Lubman DI (2008) Toluene misuse and long-term harms: A systematic review of the neuropsychological and neuroimaging literature. Neurosci Biobehavioural Rev 32:910-926. 


\section{Figure Captions}

Fig. 1 Adolescent CIT exposure attenuates weight gain throughout the exposure period (a) and results in enhanced recovery from the hypolocomotor effects of toluene inhalation (b). Body weights of CIT- $(n=41)$ compared to air-exposed rats $(n=39)$ were significantly reduced by the $6^{\text {th }}$ exposure day (Postnatal day (PN) 38), with decreased weights remaining at the cessation of the exposure period (PN 52) $(\mathbf{a} ; * * * p<0.001$, Air $v s$ CIT; Two-way RM ANOVA with Holm Sidak post hoc analysis). Enhanced recovery from the acute hypolocomotor effects of adolescent CIT exposure occurred between the initial and final toluene exposure evidenced as significantly increased horizontal plane (HP) activity in the recovery period upon the final toluene exposure $(\mathbf{b} ; * * p<0.01, * * * p<0.001$, Initial $v s$ CIT; Two-way RM ANOVA with Holm Sidak post hoc analysis). Data are mean $\pm \mathrm{SEM}$

Fig. 2 Adolescent CIT exposure has no persistent effects on motor co-ordination or motor learning as assessed in the Rotarod task. Latency to fall from Rotarod significantly increased across 3 trials for both air $(n=7)$ and CIT-exposed rats $(n=10)$ with no differences observed between groups $(\$ p<0.05$, T1 vs T2, T3; Two-way RM ANOVA with Holm Sidak post hoc analysis). Data are mean \pm SEM. (T1) Trial 1, (T2) Trial 2, (T3) Trial 3

Fig. 3 Adolescent CIT exposure does not impair spatial learning as measured in the Morris water maze (Timeline of Morris water maze protocol; a; Time points represented in the graphs are indicated by $\mathrm{b}, \mathrm{c}, \mathrm{d}$, and e). Average time to discover the submerged platform across training days did not differ between air- $(\mathrm{n}=22)$ and CIT-exposed rats $(\mathrm{n}=23)$, with both groups displaying a similar decrease in the latency to discover the submerged platform over repeated trials (b). Upon reversal, latency to discover the platform increased in both groups on the first (R1, left panel; main effect of day; Two-way RM ANOVA) but not 
second reversal (R2, right panel), with no differences observed between groups (c). In a single 1 min probe test, time spent within each quadrant did not differ between groups (d). Both air- and CIT-exposed rats displayed significantly increased time in the North East (NE) quadrant where the platform was located on day 9, and significantly decreased time in the South West (SW) quadrant where the platform was never located (d; \#\#\# $p<0.001$, NE vs NW, SE, SW; $\$ \$ \$ p<0.001$, SW $v s$ NE, NW, SE; Two-way RM ANOVA with Holm Sidak post hoc analysis). Total distance travelled within the probe test did not differ between groups (e). Data are mean of means $\pm \operatorname{SEM}(\mathbf{b}, \mathbf{c})$, or mean $\pm \operatorname{SEM}(\mathbf{d}, \mathbf{e})$. (PN) Post natal day, (REC) Recovery, (NW) North West quadrant, (SE) South East quadrant

Fig. 4 Adolescent CIT exposure results in a delay in the initial acquisition but not expression of active lever responding for 5\% sucrose with no differences in the motivation to acquire sucrose rewards between groups (Timeline of sucrose operant self-administration experiments; a; Time points represented in the graphs are indicated with b, c, d, and e). Air$(\mathrm{n}=10)$ but not CIT-exposed rats $(\mathrm{n}=8)$ significantly increased active lever responding across the first 3 operant sessions (b; \#p $<0.01$, Air day $3 v s$ day 1,2; Two-way RM ANOVA with Holm Sidak post hoc analysis). Once responding for sucrose was acquired, lever discrimination did not differ between groups (c). No significant differences were observed in progressive ratio sessions as mean total active lever responses (d) and breakpoints (defined as maximum rewards acquired) did not differ between groups (e). Data are mean of means \pm SEM. (PN) Post natal day, (REC) Recovery, (PR) Progressive ratio, (REACQ) Reacquisition sessions, (REINST) Cue-induced reinstatement session, (R1) Initial reversal sessions, (R2) Second reversal sessions

Fig. 5 Adolescent CIT exposure results in a delay of active lever responding upon cueinduced reinstatement of sucrose-seeking. An increase in total active lever responses upon cue-induced reinstatement of sucrose-seeking (REINST) compared to the final day of extinction (EXT) was observed in both air- $(n=9)$ and CIT-exposed rats $(n=8)(\mathbf{a}$; main 
effect of session; Two-way RM ANOVA), with no differences between groups. However, CIT-exposed rats displayed a significant reduction in active lever responding compared to air-exposed rats within the first 5 mins of the reinstatement session $(\mathbf{b} ; * p<0.05$, Air $v s$ CIT; Two-way RM ANOVA with Holm Sidak post hoc analysis). This appeared to be due in part to a significant increase in the latency to the initial FR3 response in CIT-exposed rats (c; $* p<0.05$; Unpaired $t$ test). Data are mean \pm SEM

Fig. 6 Operant reversal learning is initially impaired following adolescent CIT exposure. Upon the initial operant reversal session (R1) both air- $(\mathrm{n}=10)$ and CIT-exposed rats $(\mathrm{n}=$ 8) displayed a similar lack of lever discrimination with no significant differences observed between the groups (a, left panel). However, when assessing the time course of active lever responses within R1, air-exposed rats displayed a significant increase in active lever responding throughout this session (b; \# $p<0.01$, Air 5 mins vs 15 mins, 20 mins; Twoway RM ANOVA with Holm Sidak post hoc analysis), which was not observed in CITexposed rats. Upon the second operant reversal session (R2), both air- and CIT-exposed rats displayed similar active lever discrimination (a, right panel; main effect of lever; Two-way RM ANOVA). Data are mean \pm SEM 